\title{
POSITIVE OPERATORS AND APPROXIMATION IN FUNCTION SPACES ON COMPLETELY REGULAR SPACES
}

\author{
FRANCESCO ALTOMARE and SABRINA DIOMEDE
}

\author{
Received 14 January 2003
}

\begin{abstract}
We discuss the approximation properties of nets of positive linear operators acting on function spaces defined on Hausdorff completely regular spaces. A particular attention is devoted to positive operators which are defined in terms of integrals with respect to a given family of Borel measures. We present several applications which, in particular, show the advantages of such a general approach. Among other things, some new Korovkin-type theorems on function spaces on arbitrary topological spaces are obtained. Finally, a natural extension of the so-called BernsteinSchnabl operators for convex (not necessarily compact) subsets of a locally convex space is presented as well.
\end{abstract}

2000 Mathematics Subject Classification: 41A36, 47A58, 47B65.

1. Introduction. Korovkin's original theorem and its subsequent extensions and generalizations give useful criteria in order to decide whether a sequence of positive linear operators converges to the identity operator. As it is well known, these criteria involve the convergence of the sequence on special subsets of the underlying space, called Korovkin subsets.

In many concrete cases, it is also possible to give estimates of the rates of convergence in terms of quantities connected to the Korovkin subsets. We refer to [1] for more details on this subject.

On the other hand, many positive approximation processes can be defined in terms of mathematical expectation of suitable random variables taking their values into a Borel subset of some Euclidean space. In these cases, both qualitative and quantitative properties of the approximation processes can be studied by means of probabilistic methods (see [1, Section 5.2] for more details and for the relevant references).

It turns out that this approach seems to be very useful, especially for noncompact domains (for compact ones, it is indeed equivalent to the use of Korovkin-type theorems).

As a generalization of these probabilistic methods, in [11], de la Cal and Luquin proposed a general approach for studying sequences of positive linear operators defined in terms of probability measures on a given metric space or, equivalently, in terms of mathematical expectations of random variables taking their values into the same space. 
In this paper, we suggest a further more general approach for studying positive approximation processes. This approach, which is very simple and direct, generalizes the above-mentioned measure-theoretical methods and permits to extend some well-known Korovkin-type theorems as well.

The generalization we propose concerns Hausdorff topological spaces which are completely regular or, equivalently, uniformizable.

Thus this approach applies not only to metric spaces, but also to locally compact spaces, normal spaces, topological vector spaces, and so on.

The need to have some tools to study positive approximation processes on function spaces defined on possibly nonlocally compact spaces (in the locally compact case the theory is rather rich and complete, see, e.g., [1]) as well as the aim to study the approximation of continuous functions on subsets of infinitedimensional locally convex spaces were the main motivations which led us to consider such a general setting.

In the first part of the paper (Sections 3 and 4), we consider nets of positive linear operators of the form

$$
L_{i}(f)(x):=\int_{X} f d \mu_{x, i} \quad(i \in I, x \in X, f \in E),
$$

where $X$ is a Hausdorff completely regular space, $\left(\mu_{x, i}\right)_{x \in X, i \in I}$ is a family of positive Borel measures on $X$, and $E$ is the vector subspace $\bigcap_{x \in X, i \in I} \mathscr{L}^{1}\left(X, \mu_{x, i}\right)$. Moreover, the set $I$ is endowed with a directed ordering $\leq$.

We discuss under which conditions on the measures $\mu_{x, i}$ the net $\left(L_{i}\right)_{i \in I}^{\leq}$is a positive approximation process on $E$ with respect to the pointwise as well as to the uniform convergence on $X$, that is,

$$
\lim _{i \in I} \leq L_{i}(f)=f
$$

pointwise or uniformly on $X$, and we provide the relative rates of convergence.

All the results heavily depend on the family of pseudometrics which generates the topology of $X$.

In Section 5, we establish some new Korovkin-type theorems for general nets of positive linear operators (not necessarily of the form (1.1)) in the setting of completely regular spaces, extending several useful Korovkin-type theorems which have been previously established for compact spaces. Also in this case, the proofs are simple and direct.

In Section 6, we study two approximating sequences of positive linear operators acting on spaces of weakly continuous functions defined on a convex subset of some locally convex Hausdorff space.

The first one deals with the so-called Bernstein-Schnabl operators, and our results extend similar ones obtained for compact convex subsets (see [1, Chapter 6] for more details; see also [2, 3, 21, 22] for some generalization to unbounded domains). 
The other sequence of positive linear operators, which generalizes the first one, seems to be considered here for the first time, and it appears to be useful for approximating continuous functions by means of other systems of basic functions.

All the approximation formulae given throughout the paper are completed with some estimates (both pointwise and uniform) of the rate of convergence.

However, these estimates are not sharp because of the general context considered here. Better estimates can be obtained in particular settings and by using results involving other moduli of smoothness (see, e.g., [1, Chapter 5] and $[13,14,15,18])$.

2. Preliminaries and main definitions. A topological space $X$ is said to be completely regular if for every $x_{0} \in X$ and for every neighbourhood $U$ of $x_{0}$, there exists a continuous function $f: X \rightarrow \mathbb{R}, \mathbf{0} \leq f \leq \mathbf{1}$, such that

$$
f\left(x_{0}\right)=1, \quad f(x)=0 \quad \forall x \in X \backslash U .
$$

Locally compact spaces, normal spaces (and hence metric spaces), and topological vector spaces are completely regular.

A Hausdorff topological space is completely regular if and only if it is uniformizable (see [9, Chapter 2, Sections 5 and 6]) so that there exists a saturated family $\mathscr{D}$ of pseudometrics on $X$ which generates the topology of $X$.

From now on, we will fix a completely regular Hausdorff space and a saturated family $\mathscr{D}$ of pseudometrics on $X$ which generates the topology of $X$.

If $x_{0} \in X, d \in \mathscr{D}$, and $\delta>0$, we set

$$
B_{d}\left(x_{0}, \delta\right):=\left\{y \in X: d\left(x_{0}, y\right)<\delta\right\} .
$$

The family

$$
\left(B_{d}\left(x_{0}, \delta\right)\right)_{d \in \mathscr{D}, \delta>0}
$$

is a fundamental system of neighbourhoods of the point $x_{0}$.

Accordingly, a function $f: X \rightarrow \mathbb{R}$ is continuous at $x_{0} \in X$ if for every $\varepsilon>0$, there exist $d \in \mathscr{D}$ and $\delta>0$ such that

$$
\left|f(x)-f\left(x_{0}\right)\right| \leq \varepsilon \quad \forall x \in B_{d}\left(x_{0}, \delta\right) .
$$

Furthermore, $f$ is uniformly continuous if for every $\varepsilon>0$, there exist $d \in \mathscr{D}$ and $\delta>0$ such that

$$
|f(x)-f(y)| \leq \varepsilon \quad \forall x, y \in X, d(x, y) \leq \delta .
$$

Moreover, $f$ is Hölder continuous with exponent $\alpha>0$ if there exist $d \in \mathscr{D}$ and $M \geq 0$ such that

$$
|f(x)-f(y)| \leq M d(x, y)^{\alpha} \quad \forall x, y \in X .
$$


A set $H$ of real-valued functions on $X$ is equicontinuous at some point $x \in X$ if for every $\varepsilon>0$, there exist $d \in \mathscr{D}$ and $\delta>0$ such that

$$
|f(x)-f(y)| \leq \varepsilon \quad \forall f \in H, y \in X, d(x, y) \leq \delta .
$$

Furthermore, $H$ is uniformly equicontinuous on $X$ if for every $\varepsilon>0$, there exist $d \in \mathscr{D}$ and $\delta>0$ such that

$$
|f(x)-f(y)| \leq \varepsilon \quad \forall f \in H, x, y \in X, d(x, y) \leq \delta .
$$

Throughout the paper, we will use the symbols $\mathscr{F}(X, \mathbb{R}), \mathscr{B}(X, \mathbb{R}), \mathscr{C}(X, \mathbb{R})$, $\mathscr{C}_{b}(X, \mathbb{R}), U C_{b}(X, \mathbb{R})$, and $\operatorname{Lip}(X, \alpha)$ to denote the space of all real-valued functions on $X$ (resp., functions which are bounded, continuous, continuous and bounded, uniformly continuous and bounded, Hölder continuous with exponent $\alpha>0)$.

For every $f \in \mathscr{B}(X, \mathbb{R})$, we set

$$
\|f\|_{\infty}:=\sup _{x \in X}|f(x)| .
$$

As usual, the support of a function $f \in \mathscr{F}(X, \mathbb{R})$ is defined as

$$
\operatorname{Supp}(f):=\overline{\{x \in X: f(x) \neq 0\}} \text {. }
$$

We will denote by $\mathscr{K}(X, \mathbb{R})$ the space of all real-valued continuous functions whose support is a compact subset of $X$, and by $\mathscr{C}_{0}(X, \mathbb{R})$ the closure of $\mathscr{K}(X, \mathbb{R})$ in $\mathscr{C}_{b}(X, \mathbb{R})$ with respect to the uniform norm, that is,

$$
\mathscr{C}_{0}(X, \mathbb{R}):=\left\{f \in \mathscr{C}_{b}(X, \mathbb{R}): \forall \varepsilon>0, \exists g \in \mathscr{K}(X, \mathbb{R}) \text { such that }\|g-f\|_{\infty}<\varepsilon\right\} .
$$

The cone of all (positive) Borel measures on $X$ will be denoted by $\mathcal{M}^{+}(X)$. We will use the symbols $\mathcal{M}_{b}^{+}(X)$ and $\mathcal{M}_{1}^{+}(X)$ to indicate the subsets of all bounded (resp., probability) Borel measures on $X$. If $\mu \in \mu^{+}(X)$, we will denote by $\mathscr{L}^{1}(X, \mu)$ the space of all real-valued $\mu$-integrable functions on $X$.

From now on, we will fix a family

$$
\mu_{x, i} \in \mathcal{M}^{+}(X) \quad(x \in X, i \in I),
$$

where the set $I$ is endowed with a directed ordering $\leq$.

Let $E:=\bigcap_{x \in X, i \in I} \mathscr{L}^{1}\left(X, \mu_{x, i}\right)$, and for each $i \in I$ we consider the positive linear operator $L_{i}: E \rightarrow \mathscr{F}(X, \mathbb{R})$ defined by setting

$$
L_{i}(f)(x)=\int_{X} f d \mu_{x, i} \quad(f \in E, x \in X) .
$$

In the sequel, the net $\left(L_{i}\right)_{i \in I}^{\leq}$will be called the canonical net associated with the family (2.12). 
The main aim of the present paper is to study the approximation properties of the net $\left(L_{i}\right)_{i \in I}^{\leq}$. A similar investigation has been carried out in [11] when $X$ is a metric space.

However, even in this particular context, our results give new contributions to the subject.

On the other hand, these operators have been extensively studied in the case when $X$ is a real interval. We refer to [1, Section 5.2] and [12] for more details.

A probabilistic way of constructing families of measures of the form (2.12) is that of considering a probability space $(\Omega, \mathscr{F}, P)$ and a family $\left(Z_{x, i}\right)_{x \in X, i \in I}$ of random variables from $\Omega$ into $X$; then we can consider the distributions of such random variables

$$
\mu_{x, i}:=P_{Z_{x, i}} \quad(x \in X, i \in I) .
$$

In this case, the canonical net of operators associated with (2.14) can be expressed as

$$
L_{i}(f)(x):=\int_{\Omega} f \circ Z_{x, i} d P=\mathbf{E}\left(f\left(Z_{x, i}\right)\right),
$$

where the symbol $\mathbf{E}$ denotes the mathematical expectation.

In fact, by a result of Kolmogorov (see [6, Corollary 9.5]), every family $\left(\mu_{x, i}\right)_{x \in X, i \in I}$ in $\mu_{1}^{+}(X)$ is of the form (2.14).

Also notice that if a subspace $E$ of $\mathscr{F}(X, \mathbb{R})$ verifies the following integral representation property:

(P) for every positive linear form $\rho: E \rightarrow \mathbb{R}$, there exists $\mu \in \mathcal{M}^{+}(X)$ such that $E \subset \mathscr{L}^{1}(X, \mu)$ and

$$
\rho(f)=\int_{X} f d \mu \quad \forall f \in E,
$$

then every net $\left(L_{i}\right)_{i \in I}^{\leq}$of positive linear operators from $E$ into $\mathscr{F}(X, \mathbb{R})$ is the restriction of a net of operators of the form (2.13) or (2.15) provided, in addition, $\mathbf{1} \in E$ and $L_{i}(\mathbf{1})=\mathbf{1}$ for all $i \in I$.

For instance, if $X$ is a locally compact Hausdorff space, then the class of subspaces satisfying property $(\mathrm{P})$ includes the subspaces $\mathscr{K}(X, \mathbb{R}), \mathscr{C}_{0}(X, \mathbb{R})$ and, more generally, any adapted subspace of $\mathscr{C}(X, \mathbb{R})$ (see $[10$, Chapter 8 , Section 34]). Further examples for not necessarily locally compact spaces can be found in [8, Chapter 2, Theorem 2.2] or in [16].

Concrete examples of nets of positive linear operators of the form (2.13) or (2.15) are, for instance, Bernstein operators, Favard-Szász-Mirakjan operators, Baskakov operators, Gauss-Weierstrass operators, Post-Widder operators, and Stancu operators (see [1, Section 5.2] and [12] for further examples and for some relevant references).

To the same class belong the so-called Bernstein-Schnabl operators and Lototsky-Schnabl operators defined in the setting of convex compact subsets 
of locally convex spaces (see [1, Chapter 6] and [4] for details and references), as well as the operators introduced by Altomare and Carbone in [2], Altomare and Mangino in [3], and Mangino in [21].

Other examples and generalizations will be presented in Section 6.

Here we briefly discuss some conditions under which the spaces $\mathscr{C}_{b}(X, \mathbb{R})$ and $\mathscr{C}_{0}(X, \mathbb{R})$ are invariant with respect to the operators (2.13).

Fix $i \in I$. It is immediately proved that, if $f$ is any bounded function in $E$ and if

$$
\sup _{x \in X} \mu_{x, i}(X)<+\infty
$$

then $L_{i}(f)$ is bounded.

Furthermore, under assumption (2.17), $L_{i}(f) \in C_{b}(X, \mathbb{R})$ for every $f \in E \cap$ $C_{b}(X, \mathbb{R})$ if the mapping

$$
x \in X \longmapsto \mu_{x, i} \in M_{b}^{+}(X)
$$

is continuous with respect to the weak topology on $\mu_{b}^{+}(X)$.

If, in addition, $X$ is locally compact and the measures $\mu_{x, i}$ are regular, then the mapping (2.18) is continuous with respect to the weak topology if and only if $L_{i}(\mathbf{1})$ is continuous and (2.18) is continuous with respect to the vague topology on $\mathcal{M}_{b}^{+}(X)$, that is, for every $x_{0} \in X$ and $g \in \mathscr{K}(X, \mathbb{R})$,

$$
\begin{gathered}
\lim _{x \rightarrow x_{0}} \mu_{x, i}(X)=\mu_{x_{0}, i}(X), \\
\lim _{x \rightarrow x_{0}} \int_{X} g d \mu_{x, i}=\int_{X} g d \mu_{x_{0}, i}
\end{gathered}
$$

(see [7, Theorem 30.8]).

As regards the invariance of the space $\mathscr{C}_{0}(X, \mathbb{R})$, introduced in $(2.11)$, we recall that, when $X$ is locally compact, $\mathscr{C}_{0}(X, \mathbb{R})$ coincides with the space of all real continuous functions $f$ defined on $X$ which vanish at infinity; that is, for every $\varepsilon>0$, there exists a compact subset $K$ of $X$ such that $|f(x)| \leq \varepsilon$ for every $x \in X \backslash K$ (equivalently, $\lim _{x \rightarrow \infty} f(x)=0$, where $\infty$ is the point at infinity of $X$ ).

Now assume that $X$ is locally compact and the measures $\mu_{x, i}$ are regular. If (2.19) hold true and

$$
\lim _{x \rightarrow \infty} \mu_{x, i}(K)=0
$$

for every compact subset $K$ of $X$, then

$$
L_{i}(f) \in \mathscr{C}_{0}(X, \mathbb{R}) \quad \forall f \in E \cap \mathscr{C}_{0}(X, \mathbb{R}) .
$$


Indeed, for a given $\varepsilon>0$, consider two compact subsets $K_{1}$ and $K_{2}$ of $X$ such that

$$
\begin{array}{cl}
|f(t)| \leq \varepsilon & \forall t \in X \backslash K_{1}, \\
\mu_{x, i}\left(K_{1}\right) \leq \varepsilon & \forall x \in X \backslash K_{2} .
\end{array}
$$

Then, for any $x \in X \backslash K_{2}$, we have

$$
\begin{aligned}
\left|L_{i}(f)(x)\right| & \leq \int_{K_{1}}|f(t)| d \mu_{x, i}(t)+\int_{X \backslash K_{1}}|f(t)| d \mu_{x, i}(t) \\
& \leq\|f\|_{\infty} \mu_{x, i}\left(K_{1}\right)+\varepsilon \mu_{x, i}\left(X \backslash K_{1}\right) \\
& \leq\left(\|f\|_{\infty}+\sup _{x \in X} \mu_{x, i}(X)\right) \varepsilon .
\end{aligned}
$$

3. Pointwise convergence. The main aim of this section is to analyze the pointwise approximation properties of the net (2.13). At the same time, estimates of the rates of convergence will also be given.

We again fix a completely regular Hausdorff space $X$ and a saturated family $\mathscr{D}$ of pseudometrics on $X$ which generates the topology of $X$.

We will consider a family $\left(\mu_{x, i}\right)_{x \in X, i \in I}$ and the canonical net of operators (2.13) defined on $E:=\bigcap_{x \in X, i \in I} \mathscr{L}^{1}\left(X, \mu_{x, i}\right)$.

We have the following result.

THEOREM 3.1. Let $x \in X$ and assume that

$$
\lim _{i \in I}^{\leq} \mu_{x, i}(X)=1
$$

Then, the following statements hold true.

(1) If for every $d \in \mathscr{D}$ and $\delta>0$,

$$
\lim _{i \in I} \leq \mu_{x, i}\left(X \backslash B_{d}(x, \delta)\right)=0,
$$

then for every function $f \in E$ which is bounded and continuous at $x$,

$$
\lim _{i \in I}^{\leq} L_{i}(f)(x)=f(x) .
$$

Moreover, if $H$ is an equibounded subset of $E \cap \mathscr{B}(X, \mathbb{R})$, that is, $\sup _{f \in H}\|f\|_{\infty}<$ $+\infty$, which is equicontinuous at $x$, then (3.3) holds true uniformly with respect to $f \in H$.

(2) If there exists $\beta>0$ such that for each $d \in \mathscr{D}$,

$$
\lim _{i \in I} \leq \int_{X} d(x, y)^{\beta} d \mu_{x, i}(y)=0,
$$

then (3.3) holds true for every $f \in E \cap \operatorname{Lip}(X, \alpha)$ with $0<\alpha \leq \beta$. 
Proof. (1) Consider a bounded function $f \in E$ and assume that it is continuous at $x \in X$; for $\varepsilon>0$, there exist $\delta>0$ and $d \in \mathscr{D}$ such that

$$
|f(x)-f(y)| \leq \varepsilon \quad \forall y \in B_{d}(x, \delta) .
$$

Moreover, by virtue of (3.1) and (3.2), there exists $i_{0} \in I$ such that for all $i \in I$, $i \geq i_{0}$, one has

$$
\begin{gathered}
\left|\mu_{x, i}(X)-1\right| \leq \varepsilon, \\
\mu_{x, i}\left(X \backslash B_{d}(x, \delta)\right) \leq \varepsilon .
\end{gathered}
$$

Therefore, for any $i \geq i_{0}$,

$$
\begin{aligned}
\left|L_{i}(f)(x)-f(x)\right| \leq & \left|L_{i}(f)(x)-\mu_{x, i}(X) f(x)\right|+\left|\mu_{x, i}(X) f(x)-f(x)\right| \\
\leq & \int_{X}|f(y)-f(x)| d \mu_{x, i}(y)+\left|\mu_{x, i}(X)-1\right||f(x)| \\
\leq & \int_{B_{d}(x, \delta)}|f(y)-f(x)| d \mu_{x, i}(y) \\
& +\int_{X \backslash B_{d}(x, \delta)}|f(y)-f(x)| d \mu_{x, i}(y)+\varepsilon|f(x)| \\
\leq & \varepsilon \mu_{x, i}(X)+2\|f\|_{\infty} \mu_{x, i}\left(X \backslash B_{d}(x, \delta)\right)+\varepsilon|f(x)| \\
\leq & \varepsilon\left(1+\varepsilon+3\|f\|_{\infty}\right) .
\end{aligned}
$$

The second part of the statement may be proved in a similar way.

(2) Consider $f \in E \cap \operatorname{Lip}(X, \alpha)$ for some $\alpha \in] 0, \beta]$; then there exist $M \geq 0$ and $d \in \mathscr{D}$ such that

$$
|f(x)-f(y)| \leq M d(x, y)^{\alpha} \quad \forall x, y \in X
$$

Fix $\varepsilon>0$ and set $\delta:=\varepsilon^{1 / \alpha}$. By hypothesis, there is an index $i_{0} \in I$ such that, for all $i \in I, i \geq i_{0}$, one has

$$
\begin{gathered}
\left|\mu_{x, i}(X)-1\right| \leq \varepsilon, \\
\int_{X} d(x, y)^{\beta} d \mu_{x, i}(y) \leq \varepsilon \delta^{\beta-\alpha} .
\end{gathered}
$$

Now observe that, for every $y \in X, d(x, y) \geq \delta$, we have

$$
|f(x)-f(y)| \leq M d(x, y)^{\alpha} \frac{d(x, y)^{\beta-\alpha}}{\delta^{\beta-\alpha}}=\frac{M}{\delta^{\beta-\alpha}} d(x, y)^{\beta}
$$


therefore, for $i \in I, i \geq i_{0}$, one has

$$
\begin{aligned}
\left|L_{i}(f)(x)-f(x)\right| \\
\leq|| \mu_{x, i}(X)-1|| f(x)\left|+\int_{B_{d}(x, \delta)}\right| f(y)-f(x) \mid d \mu_{x, i}(y) \\
\quad+\int_{X \backslash B_{d}(x, \delta)}|f(y)-f(x)| d \mu_{x, i}(y) \\
\leq \varepsilon|f(x)|+M \varepsilon \mu_{x, i}(X)+\frac{M}{\delta^{\beta-\alpha}} \int_{X \backslash B_{d}(x, \delta)} d(x, y)^{\beta} d \mu_{x, i}(y) \\
\leq \varepsilon(|f(x)|+M(1+\varepsilon)+M \varepsilon) .
\end{aligned}
$$

REMARK 3.2. (1) Condition (3.1) is also necessary provided $\mathbf{1} \in E$. Indeed, it corresponds to formula (3.3) in the particular case in which $f=\mathbf{1}$.

(2) If the measures $\mu_{x, i}$ are the distributions of suitable random variables $Z_{x, i}$ as in (2.14), then condition (3.2) means that

$$
\lim _{i \in I}^{\leq} Z_{x, i}=Z_{x}
$$

stochastically, that is, for every $d \in \mathscr{D}$ and $\delta>0$,

$$
\lim _{i \in I} \leq P\left\{d\left(Z_{x, i}, Z_{x}\right) \geq \delta\right\}=0 .
$$

Here $Z_{x}: \Omega \mapsto X$ denotes the random variable which takes the constant value $x$.

(3) Condition (3.4) implies (3.2). Indeed, by the Chebychev-Markov inequality (see [7, Lemma 20.1]), for any $d \in \mathscr{D}$, we have

$$
\mu_{x, i}\left(X \backslash B_{d}(x, \delta)\right)=\mu_{x, i}\{d(x, \cdot) \geq \delta\} \leq \frac{1}{\delta^{\beta}} \int_{X} d(x, \cdot)^{\beta} d \mu_{x, i} .
$$

(4) If $X$ is bounded, that is, for every $d \in \mathscr{D}$,

$$
\operatorname{diam}_{d}(X):=\sup _{x, y \in X} d(x, y)<+\infty,
$$

then conditions (3.1) and (3.2) imply condition (3.4) for every $\beta>0$ and $d \in \mathscr{D}$. Indeed, for a given $\delta>0$, we have

$$
\begin{aligned}
\int_{X} d(x, \cdot)^{\beta} d \mu_{x, i} & =\int_{B_{d}(x, \delta)} d(x, \cdot)^{\beta} d \mu_{x, i}+\int_{X \backslash B_{d}(x, \delta)} d(x, \cdot)^{\beta} d \mu_{x, i} \\
& \leq \delta^{\beta} \mu_{x, i}(X)+\operatorname{diam}_{d}(X)^{\beta} \mu_{x, i}\left(X \backslash B_{d}(x, \delta)\right)
\end{aligned}
$$

and so (3.4) follows since $\delta$ was arbitrarily chosen.

We will complete the previous results about pointwise convergence by providing some estimates of the rate of convergence.

However, these estimates (as well as the uniform ones we will present in the next sections) are not sharp because of the generality of the context considered 
here. Better estimates can be obtained in particular settings (especially in real intervals) by using results involving other moduli of smoothness (see, e.g., [1, Chapter 5] and [13, 14, 15, 18]).

We will now introduce the following generalized moduli of continuity.

For every $f \in \mathscr{F}(X, \mathbb{R}), \delta>0$, and $x \in X$, we set

$$
\begin{gathered}
\omega_{d, x}(f, \delta):=\sup _{\substack{y \in X \\
d(x, y) \leq \delta}}|f(x)-f(y)| \in \mathbb{R} \cup\{+\infty\}, \\
\omega_{d}(f, \delta):=\sup _{\substack{x, y \in X \\
d(x, y) \leq \delta}}|f(x)-f(y)| \in \mathbb{R} \cup\{+\infty\} .
\end{gathered}
$$

Then we have the following result.

Proposition 3.3. Let $x \in X$. Then

(1) if $d \in \mathscr{D}, \delta>0, \beta>0, i \in I$, and $f \in E \cap \mathscr{B}(X, \mathbb{R})$, then

$$
\begin{aligned}
\left|L_{i}(f)(x)-f(x)\right| \leq & \omega_{d, x}(f, \delta) \mu_{x, i}(X)+2\|f\|_{\infty} \mu_{x, i}\left(X \backslash B_{d}(x, \delta)\right) \\
& +|f(x)|\left|1-\mu_{x, i}(X)\right| \\
\leq & \omega_{d, x}(f, \delta)+2 \frac{\|f\|_{\infty}}{\delta^{\beta}} \int_{X} d(x, \cdot)^{\beta} d \mu_{x, i} \\
& +|f(x)|\left|1-\mu_{x, i}(X)\right|
\end{aligned}
$$

(2) for every $\alpha>0$ and for all $f \in E \cap \operatorname{Lip}(X, \alpha)$, there are $M \geq 0$ and $d \in \mathscr{D}$ such that for every $\beta \geq \alpha, \delta>0, x \in X$, and $i \in I$,

$$
\begin{aligned}
\left|L_{i}(f)(x)-f(x)\right| \leq & M \delta^{\alpha} \mu_{x, i}(X)+\frac{M}{\delta^{\beta-\alpha}} \int_{X} d(x, \cdot)^{\beta} d \mu_{x, i} \\
& +|f(x)|\left|1-\mu_{x, i}(X)\right|
\end{aligned}
$$

In particular, for $\delta:=\left(\int_{X} d(x, \cdot)^{\beta} d \mu_{x, i}\right)^{1 / \beta}$ (which can always be assumed to be strictly positive),

$$
\begin{aligned}
\left|L_{i}(f)(x)-f(x)\right| \leq & M\left(\mu_{x, i}(X)+1\right)\left(\int_{X} d(x, \cdot)^{\beta} d \mu_{x, i}\right)^{\alpha / \beta} \\
& +|f(x)|\left|1-\mu_{x, i}(X)\right| .
\end{aligned}
$$

Proof. It is enough to refer to the same inequalities and arguments used in the proof of Theorem 3.1 (see also Remark 3.2(3)).

4. Uniform convergence. Before examining the uniform convergence of the approximation process given by (2.13), we present some preliminary results which can be proved in the same way as for metric spaces.

LEMMA 4.1. The space $\mathscr{C}_{0}(X, \mathbb{R})$ is contained in the space $U C_{b}(X, \mathbb{R})$. 
LEMMA 4.2. Let $K$ be a compact subset of $X$ and $\left(U_{i}\right)_{i \in I}$ a family of open subsets of $X$ such that

$$
K \subset \bigcup_{i \in I} U_{i}
$$

Then there are $\delta>0$ and $d \in \mathscr{D}$ such that, for any $x \in K$, there exists $i \in I$ for which $B_{d}(x, \delta) \subset U_{i}$.

Making use of Lemmas 4.1 and 4.2, we deduce the following result.

Proposition 4.3. Let $H$ be an equicontinuous subset of $\mathscr{C}_{b}(X, \mathbb{R})$ and assume that it is equibounded, that is, $M:=\sup _{f \in H}\|f\|_{\infty}<+\infty$; then for any $u \in \mathscr{K}(X, \mathbb{R})$, the set

$$
u H:=\{u f: f \in H\}
$$

is uniformly equicontinuous.

In particular, if $X$ is compact, then $H$ itself is uniformly equicontinuous.

Proof. Set $\varepsilon>0$; by Lemma 4.1, $u$ is uniformly continuous, and therefore there are $\delta_{1}>0$ and $d_{1} \in \mathscr{D}$ so that

$$
|u(x)-u(y)| \leq \varepsilon \quad \forall x, y \in X, d_{1}(x, y) \leq \delta_{1} .
$$

For each $x \in \operatorname{Supp}(u)$, by the equicontinuity of $H$, there exist $\delta(x)>0$ and $d_{x} \in \mathscr{D}$ for which

$$
|f(x)-f(y)| \leq \varepsilon \quad\left(f \in H, y \in B_{d_{x}}(x, \delta(x))\right) .
$$

Since

$$
\operatorname{Supp}(u) \subset \bigcup_{x \in \operatorname{Supp}(u)} B_{d_{x}}(x, \delta(x)),
$$

by virtue of Lemma 4.2, there exist $\delta_{2}>0$ and $d_{2} \in \mathscr{D}$ such that, for any $x \in$ $\operatorname{Supp}(u)$, there is some $z \in \operatorname{Supp}(u)$ so that

$$
B_{d_{2}}\left(x, \delta_{2}\right) \subset B_{d_{z}}(z, \delta(z))
$$

Now, set

$$
\delta:=\min \left\{\delta_{1}, \delta_{2}\right\}, \quad d:=\sup \left\{d_{1}, d_{2}\right\} \in \mathscr{D},
$$

and consider $f \in H$ and $x, y \in X$ such that $d(x, y) \leq \delta$. In case both $x$ and $y$ do not belong to $\operatorname{Supp}(u)$, then, of course,

$$
|f(x) u(x)-f(y) u(y)|=0 \leq \varepsilon .
$$


If $\operatorname{Supp}(u)$ contains one (or both) of the points $x$ and $y$, say, for instance, $x$, then

$$
\begin{aligned}
& |f(x) u(x)-f(y) u(y)| \\
& \quad \leq|f(x) u(x)-f(x) u(y)|+|f(x) u(y)-f(y) u(y)| \\
& \quad \leq|f(x)||u(x)-u(y)|+|u(y)||f(x)-f(y)| \\
& \quad \leq M|u(x)-u(y)|+\|u\|_{\infty}|f(x)-f(y)| .
\end{aligned}
$$

Moreover, there is some $z \in \operatorname{Supp}(u)$ as required in (4.6). As $d_{2}(x, y) \leq d(x, y)$ $\leq \delta \leq \delta_{2}$, we have

$$
y \in B_{d_{2}}\left(x, \delta_{2}\right) \subset B_{d_{z}}(z, \delta(z)),
$$

and so $y, x \in B_{d_{z}}(z, \delta(z))$; thus, by virtue of (4.4),

$$
|f(x)-f(y)| \leq 2 \varepsilon .
$$

Moreover, since $d_{1}(x, y) \leq d(x, y) \leq \delta \leq \delta_{1}$,

$$
|u(x)-u(y)| \leq \varepsilon,
$$

because of (4.3). This last inequality, joined with (4.11) and inserted in (4.9), leads to the assertion.

Finally, if $X$ is compact, by applying the previous result to $u=\mathbf{1}$, we get the last statement.

Now we recall that the space $X$ is said to be precompact, or totally bounded, if for every $\varepsilon>0$ and $d \in \mathscr{D}$, there exist finitely many subsets $X_{1}, \ldots, X_{n}$ of $X$ such that

$$
X=\bigcup_{i=1}^{n} X_{i}, \quad \operatorname{diam}_{d}\left(X_{i}\right) \leq \varepsilon
$$

for every $i=1, \ldots, n$ (see, e.g., [9, page 83]).

If $X$ is precompact, then it is bounded. Moreover, if $X$ is compact, then it is precompact.

We can now state the main results of this section.

THEOREM 4.4. Assume that

$$
\begin{aligned}
\lim _{i \in I}^{\leq} \mu_{x, i}(X)=1 & \text { uniformly with respect to } x \in X, \\
\lim _{i \in I}^{\leq} \mu_{x, i}\left(X \backslash B_{d}(x, \delta)\right)=0 & \text { uniformly with respect to } x \in X,
\end{aligned}
$$

for every $d \in \mathscr{D}$ and $\delta>0$.

The following statements hold true.

(1) For every $f \in E \cap U C_{b}(X, \mathbb{R}), \lim _{i \in I}^{\leq} L_{i}(f)=f$ uniformly on $X$. 
(2) If $H$ is an equibounded and uniformly equicontinuous subset $H$ of $E \cap$ $C_{b}(X, \mathbb{R})$, then $\lim _{i \in I}^{\leq} L_{i}(f)=f$ uniformly on $X$ and uniformly with respect to $f \in H$.

(3) If $X$ is bounded, then statement (2) implies (4.14) and (4.15).

(4) If $X$ is precompact, then statement (1) implies (4.14) and (4.15).

Proof. (1) Consider $f \in U C_{b}(X, \mathbb{R})$ and fix $\varepsilon>0$; then there are $\delta>0$ and $d \in \mathscr{D}$ so that

$$
|f(x)-f(y)|<\varepsilon \quad \forall x, y \in X, d(x, y) \leq \delta,
$$

and there exists an index $i_{0} \in I$ such that

$$
M:=\sup _{i \geq i_{0}} \sup _{x \in X} \mu_{x, i}(X)<+\infty
$$

and for any $i \geq i_{0}$,

$$
\begin{array}{rr}
\mu_{x, i}\left(X \backslash B_{d}(x, \delta)\right) \leq \varepsilon & \forall x \in X, \\
\left|\mu_{x, i}(X)-1\right|<\varepsilon & \forall x \in X .
\end{array}
$$

Then, given $x \in X$ and $i \geq i_{0}$, one has

$$
\begin{aligned}
\left|L_{i}(f)(x)-f(x)\right| \\
\leq\left|\mu_{x, i}(X)-1\right||f(x)|+\int_{B_{d}(x, \delta)}|f(y)-f(x)| d \mu_{x, i}(y) \\
\quad+\int_{X \backslash B_{d}(x, \delta)}|f(y)-f(x)| d \mu_{x, i}(y) \\
\leq \varepsilon\|f\|_{\infty}+\varepsilon \mu_{x, i}(X)+2\|f\|_{\infty} \mu_{x, i}\left(X \backslash B_{d}(x, \delta)\right) \\
\leq \varepsilon\left(M+3\|f\|_{\infty}\right) .
\end{aligned}
$$

With a similar reasoning, one can also show part (2).

Now assume that $X$ is bounded and that statement (2) holds true. Clearly, we get (4.14) by simply applying assertion (2) to $H:=\{\mathbf{1}\}$.

Moreover, according to Remark 3.2(3), to obtain (4.15), it suffices to show that $\lim _{i \in I}^{\leq} \int_{X} d(x, \cdot) d \mu_{x, i}=0$ uniformly with respect to $x \in X$, for every $d \in \mathscr{D}$, that is,

$$
\lim _{i \in I}^{\leq} L_{i}(d(x, \cdot))(x)=0 \quad \text { uniformly with respect to } x \in X \text {. }
$$

This last limit relation will follow from statement (2) if we show that the set

$$
H:=\{d(x, \cdot): x \in X\}
$$

is equibounded and uniformly equicontinuous. 
Clearly, $H$ is equibounded since $M:=\operatorname{diam}_{d}(X)<+\infty$. On the other hand, for every $x, y, z \in X$, we get

$$
|d(x, y)-d(x, z)| \leq d(y, z)
$$

and hence $H$ is uniformly equicontinuous.

Finally, assume that $X$ is precompact and that statement (1) holds true.

Clearly, (4.14) is straightforward. To obtain (4.15), we will again prove (4.20).

For given $d \in \mathscr{D}$ and $\varepsilon>0$, choose finitely many subsets $X_{1}, \ldots, X_{n}$ of $X$ such that $X=\bigcup_{p=1}^{n} X_{p}$ and $\operatorname{diam}_{d}\left(X_{p}\right) \leq \varepsilon$ for all $p=1, \ldots, n$.

Choose a point $x_{p} \in X_{p}$ for any $p=1, \ldots, n$. By the above reasoning, we know that each $d\left(x_{p}, \cdot\right) \in E \cap U C_{b}(X, \mathbb{R})$; and hence from statement (1), it follows that there exist $M \geq 0$ and $i_{0} \in I$ such that, for $i \geq i_{0}, p=1, \ldots, n$, and $x \in X$, we have

$$
\left|L_{i}\left(d\left(x_{p}, \cdot\right)\right)(x)-d\left(x_{p}, x\right)\right| \leq \varepsilon, \quad \mu_{x, i}(X) \leq M .
$$

Therefore, if $i \geq i_{0}$ and $x \in X$, after choosing $p=1, \ldots, n$ such that $x \in X_{p}$, we obtain

$$
\int_{X} d\left(x_{p}, \cdot\right) d \mu_{x, i} \leq\left|L_{i}\left(d\left(x_{p}, \cdot\right)\right)(x)-d\left(x_{p}, x\right)\right|+d\left(x_{p}, x\right) \leq 2 \varepsilon,
$$

and hence

$$
\begin{aligned}
\int_{X} d(x, \cdot) d \mu_{x, i} & \leq \int_{X}\left|d(x, \cdot)-d\left(x_{p}, \cdot\right)\right| d \mu_{x, i}+\int_{X} d\left(x_{p}, \cdot\right) d \mu_{x, i} \\
& \leq \int_{X} d\left(x_{p}, x\right) d \mu_{x, i}+2 \varepsilon \leq(2+M) \varepsilon .
\end{aligned}
$$

This completes the proof of Theorem 4.4.

When $X$ is locally compact, we can say something about the convergence of the net (2.13) on not necessarily uniformly continuous functions.

THEOREM 4.5. Assume that $X$ is locally compact and that properties (4.14) and (4.15) hold true. Then

(1) for every $f \in E \cap C_{b}(X, \mathbb{R}), \lim _{i \in I}^{\leq} L_{i}(f)=f$ uniformly on compact subsets of $X$;

(2) if $H$ is an equibounded and equicontinuous subset $H$ of $E \cap C_{b}(X, \mathbb{R})$, then $\lim _{i \in I}^{\leq} L_{i}(f)=f$ uniformly on compact subsets of $X$ and uniformly with respect to $f \in H$.

Proof. (1) Let $f \in E \cap \mathscr{C}_{b}(X, \mathbb{R})$, let $K$ be a compact subset of $X$, and let choose $\varepsilon \in] 0,1[$. By virtue of Urysohn's theorem, there exists $u \in \mathscr{K}(X, \mathbb{R})$ so 
that $\mathbf{0} \leq u \leq \mathbf{1}$ and

$$
1-\varepsilon \leq u \text { on } K \text {. }
$$

We remark that both $u, u f \in U C_{b}(X, \mathbb{R})$ (see Lemma 4.1) and thus, applying Theorem 4.4(1), there is $i_{0} \in I$ so that, for all $i \geq i_{0}$ and $x \in X$,

$$
\begin{gathered}
\left|L_{i}(1-u)(x)-(1-u)(x)\right| \leq \varepsilon, \\
\left|L_{i}(f u)(x)-f(x) u(x)\right| \leq \varepsilon .
\end{gathered}
$$

Observe, in particular, that for any $y \in K$, one has

$$
\int_{X}(\mathbf{1}-u) d \mu_{y, i}=L_{i}(\mathbf{1}-u)(y) \leq 2 \varepsilon \quad\left(i \geq i_{0}\right) .
$$

Let $x \in K$; then for any $i \geq i_{0}$,

$$
\begin{aligned}
\left|L_{i}(f)(x)-f(x)\right| \leq & \left|\int_{X} f d \mu_{x, i}-\int_{X} f u d \mu_{x, i}\right| \\
& +\left|\int_{X} f u d \mu_{x, i}-f(x) u(x)\right|+|f(x) u(x)-f(x)| \\
\leq & \|f\|_{\infty} \int_{X}(1-u) d \mu_{x, i} \\
& +\left|L_{i}(f u)(x)-f(x) u(x)\right|+\|f\|_{\infty}(1-u(x)) \\
\leq & \left(3\|f\|_{\infty}+1\right) \varepsilon .
\end{aligned}
$$

(2) It suffices to follow the scheme of the proof of part (1), recalling that, by Proposition 4.3 , for any $u \in \mathscr{K}(X, \mathbb{R})$, the set $u H$ is uniformly equicontinuous.

REMARK 4.6. (1) If the $\mu_{x, i}$ are the distributions of some random variables $Z_{x, i}$, then by arguing as in Remark 3.2, one can see that condition (4.15) means that $\lim _{i \in I}^{\leq} Z_{x, i}=Z_{x}$ stochastically uniformly with respect to $x \in X$, that is, for every $d \in \mathscr{D}$ and $\delta>0$,

$$
\lim _{i \in I}^{\leq} \sup _{x \in X} P\left\{d\left(Z_{x, i}, Z_{x}\right) \geq \delta\right\}=0
$$

Moreover, (4.15) holds true if there exists $\beta>0$ such that, for every $d \in \mathscr{D}$,

$$
\lim _{i \in I} \leq \int_{X} d(x, \cdot)^{\beta} d \mu_{x, i}=0 \quad \text { uniformly with respect to } x \in X \text {. }
$$

Conversely, if $X$ is bounded, then (4.14) and (4.15) imply (4.31) for every $\beta>0$ (see Remark 3.2(4)). 
(2) From the proof of Theorem 4.4 and from Chebychev-Markov inequality (see Remark 3.2(3)), it follows that if $f \in E \cap U C_{b}(X, \mathbb{R})$, then for every $d \in \mathscr{D}$, $\delta>0$, and $\beta>0$, one gets

$$
\begin{aligned}
\sup _{x \in X}\left|L_{i}(f)(x)-f(x)\right| \leq & \|f\|_{\infty} \sup _{x \in X}\left|\mu_{x, i}(X)-1\right|+\omega_{d}(f, \delta) \sup _{x \in X} \mu_{x, i}(X) \\
& +2\|f\|_{\infty} \sup _{x \in X} \mu_{x, i}\left(X \backslash B_{d}(x, \delta)\right) \\
\leq & \|f\|_{\infty} \sup _{x \in X}\left|\mu_{x, i}(X)-1\right|+\omega_{d}(f, \delta) \sup _{x \in X} \mu_{x, i}(X) \\
& +\frac{2\|f\|_{\infty}}{\delta^{\beta}} \sup _{x \in X} \int_{X} d(x, \cdot)^{\beta} d \mu_{x, i}
\end{aligned}
$$

for every $i \in I$. Furthermore, there exists $i_{0} \in I$ depending on $d \in \mathscr{D}$ and $\delta>0$ (resp., on $d \in \mathscr{D}$ and $\beta>0$ provided (4.15) is replaced by (4.31)) such that, for any $i \in I, i \geq i_{0}$, all the suprema indicated in (4.32) are finite.

Finally, Proposition 3.3(2) implies that, if $f \in E \cap C_{b}(X, \mathbb{R}) \cap \operatorname{Lip}(X, \alpha), 0<\alpha$, then there exist $M \geq 0$ and $d \in \mathscr{D}$ such that, for any $\delta>0$ and $\beta \geq \alpha$, we have

$$
\begin{aligned}
\sup _{x \in X}\left|L_{i}(f)(x)-f(x)\right| \leq & \|f\|_{\infty} \sup _{x \in X}\left|\mu_{x, i}(X)-1\right|+M \delta^{\alpha} \sup _{x \in X} \mu_{x, i}(X) \\
& +\frac{M}{\delta^{\beta-\alpha}} \sup _{x \in X} \int_{X} d(x, \cdot)^{\beta} d \mu_{x, i}
\end{aligned}
$$

for every $i \in I$. Furthermore, if (4.31) holds true for some $\beta \geq \alpha$ and $d \in \mathscr{D}$, then there exists $i_{0} \in I$ depending on $d$ and $\beta$ such that for any $i \in I, i \geq i_{0}$, all the suprema indicated in (4.33) are finite.

In particular, for $i \geq i_{0}$ and $\delta:=\left(\sup _{x \in X} \int_{X} d(x, \cdot)^{\beta} d \mu_{x, i}\right)^{1 / \beta}$ (which we may always assume to be strictly positive), we get

$$
\begin{aligned}
\left\|L_{i}(f)-f\right\|_{\infty} \leq & \|f\|_{\infty} \sup _{x \in X}\left|\mu_{x, i}(X)-1\right| \\
& +M\left(\sup _{x \in X} \mu_{x, i}(X)+1\right)\left(\sup _{x \in X} \int_{X} d(x, \cdot)^{\beta} d \mu_{x, i}\right)^{\alpha / \beta} .
\end{aligned}
$$

5. Some Korovkin-type theorems. In this section, by using some simple methods similar to those used in the previous sections, we will prove some Korovkin-type theorems. Notice also that the next results concern arbitrary nets of positive linear operators (not necessarily of the form (2.13)).

Again we fix a completely regular Hausdorff space $X$ and a saturated family $\mathscr{D}$ of pseudometrics on $X$ which generates the topology of $X$.

The next result generalizes [5, Theorem 3], which was established for compact metric spaces with different methods.

THEOREM 5.1. Let $E$ be a linear subspace of $\mathscr{F}(X, \mathbb{R})$ such that $\mathbf{1} \in$ E. Also assume that there exist $\beta>0$ and a family $\left(\psi_{d, x}\right)_{x \in X, d \in \mathscr{D}}$ in $E$ such that $d(x, \cdot)^{\beta}$ $\leq \psi_{d, x}$ for every $x \in X$ and $d \in \mathscr{D}$. Let $\left(L_{i}\right)_{i \in I}^{\leq}$be a net of positive linear operators from $E$ into $\mathscr{F}(X, \mathbb{R})$ such that 
(i) $\lim _{i \in I}^{\leq} L_{i}(\mathbf{1})=1$ uniformly (resp., pointwise) on $X$;

(ii) for each $d \in \mathscr{D}$,

$$
\lim _{i \in I}^{\leq} L_{i}\left(\psi_{d, x}\right)(x)=0
$$

uniformly with respect to $x \in X$ (resp., for every $x \in X$ ).

Then

$$
\lim _{i \in I}^{\leq} L_{i}(f)=f
$$

uniformly on $X$ for every $f \in E \cap U C_{b}(X, \mathbb{R})$, respectively,

$$
\lim _{i \in I}^{\leq} L_{i}(f)=f
$$

pointwise on $X$ for every $f \in E \cap \mathscr{C}_{b}(X, \mathbb{R})$. Moreover, if $X$ is locally compact,

$$
\lim _{i \in I} \leq L_{i}(f)=f
$$

uniformly on compact subsets of $X$, for every $f \in E \cap \mathscr{C}_{b}(X, \mathbb{R})$.

Finally, the limits (5.2) and (5.4) (resp., the limit (5.3)) hold uniformly with respect to $f \in H$ provided $H$ is an equibounded and uniformly equicontinuous (resp., equicontinuous) subset of $E \cap \mathscr{C}_{b}(X, \mathbb{R})$.

Proof. Let $f \in E \cap U C_{b}(X, \mathbb{R})$ and fix $\varepsilon>0$; then there exist $d \in \mathscr{D}$ and $\delta>0$ such that

$$
|f(x)-f(y)| \leq \varepsilon \quad \forall x, y \in X, d(x, y) \leq \delta .
$$

On the other hand, if $x, y \in X$ and $d(x, y) \geq \delta$, we have

$$
|f(x)-f(y)| \leq 2\|f\|_{\infty} \leq 2\|f\|_{\infty} \frac{d(x, y)^{\beta}}{\delta^{\beta}} .
$$

In any case, for any $x, y \in X$, we get

$$
|f(x)-f(y)| \leq \varepsilon+2\|f\|_{\infty} \frac{d(x, y)^{\beta}}{\delta^{\beta}},
$$

that is,

$$
|f(x) \mathbf{1}-f| \leq \varepsilon \mathbf{1}+2 \frac{\|f\|_{\infty}}{\delta^{\beta}} d(x, \cdot)^{\beta} \leq \varepsilon \mathbf{1}+2 \frac{\|f\|_{\infty}}{\delta^{\beta}} \psi_{d, x} .
$$

On the other hand, there exist $i_{0} \in I$ and $M \geq 0$ such that, for each $i \geq i_{0}$ and $x \in X$, we have

$$
\left|L_{i}(\mathbf{1})(x)\right| \leq M, \quad L_{i}\left(\psi_{d, x}\right)(x) \leq \varepsilon \delta^{\beta}, \quad\left|L_{i}(\mathbf{1})(x)-1\right| \leq \varepsilon .
$$


Accordingly, since

$$
\left|L_{i}(f)-f(x) L_{i}(\mathbf{1})\right| \leq \varepsilon L_{i}(\mathbf{1})+2 \frac{\|f\|_{\infty}}{\delta^{\beta}} L_{i}\left(\psi_{d, x}\right),
$$

we get

$$
\begin{aligned}
\left|L_{i}(f)(x)-f(x)\right| \leq & \left|L_{i}(f)(x)-f(x) L_{i}(\mathbf{1})(x)\right| \\
& +|f(x)|\left|L_{i}(\mathbf{1})(x)-1\right| \\
\leq & \varepsilon L_{i}(\mathbf{1})(x)+2 \frac{\|f\|_{\infty}}{\delta^{\beta}} L_{i}\left(\psi_{d, x}\right)(x) \\
& +\|f\|_{\infty}\left|L_{i}(\mathbf{1})(x)-1\right| \leq\left(M+3\|f\|_{\infty}\right) \varepsilon .
\end{aligned}
$$

A similar proof runs provided $f \in H$, with $H$ being equibounded and uniformly equicontinuous.

The proof of the other statements is similar to the one of Theorem 4.5.

REMARK 5.2. (1) As the above proof shows, for $f \in E \cap C_{b}(X, \mathbb{R}), i \in I, x \in X$, $d \in \mathscr{D}$, and $\delta>0$, we have (see (3.18))

$$
\begin{aligned}
\left|L_{i}(f)(x)-f(x)\right| \leq & |f(x)|\left|L_{i}(\mathbf{1})(x)-1\right| \\
& +\omega_{d, x}(f, \delta) L_{i}(\mathbf{1})(x)+2 \frac{\|f\|_{\infty}}{\delta^{\beta}} L_{i}\left(\psi_{d, x}\right)(x)
\end{aligned}
$$

and, if $f \in U C_{b}(X, \mathbb{R})$,

$$
\begin{aligned}
\left\|L_{i}(f)-f\right\|_{\infty} \leq & \|f\|_{\infty}\left\|L_{i}(\mathbf{1})-\mathbf{1}\right\|_{\infty} \\
& +\omega_{d}(f, \delta)\left\|L_{i}(\mathbf{1})\right\|_{\infty}+2 \frac{\|f\|_{\infty}}{\delta^{\beta}} \sup _{x \in X} L_{i}\left(\psi_{d, x}\right)(x) .
\end{aligned}
$$

Moreover, if $f \in E \cap \mathscr{C}_{b}(X, \mathbb{R}) \cap \operatorname{Lip}(X, \alpha)$, with $0<\alpha \leq \beta$, then there exist $M \geq 0$ and $d \in \mathscr{D}$ such that, for every $i \in I$ and $\delta>0$, one has

$$
\begin{aligned}
\left\|L_{i}(f)-f\right\|_{\infty} \leq & \|f\|_{\infty}\left\|L_{i}(\mathbf{1})-\mathbf{1}\right\|_{\infty} \\
& +M \delta^{\alpha}\left\|L_{i}(\mathbf{1})\right\|_{\infty}+\frac{M}{\delta^{\beta-\alpha}} \sup _{x \in X} L_{i}\left(\psi_{d, x}\right)(x)
\end{aligned}
$$

(see Remark 4.6(2)).

In particular,

$$
\begin{aligned}
\left\|L_{i}(f)-f\right\|_{\infty} \leq & \|f\|_{\infty}\left\|L_{i}(\mathbf{1})-\mathbf{1}\right\|_{\infty} \\
& +M\left(\left\|L_{i}(\mathbf{1})\right\|_{\infty}+1\right)\left(\sup _{x \in X} L_{i}\left(\psi_{d, x}\right)(x)\right)^{\alpha / \beta} .
\end{aligned}
$$

Indeed, set $\delta_{0}:=\left(\sup _{x \in X} L_{i}\left(\psi_{d, x}\right)(x)\right)^{1 / \beta}$. If $\delta_{0}=0$, then (5.15) follows from (5.14) by letting $\delta \rightarrow 0$; if, instead, $\delta_{0}>0$, then (5.15) is obtained applying (5.14) to $\delta=\delta_{0}$. 
(2) If $d(x, \cdot)^{\beta} \in E$ for every $x \in X$ and $d \in \mathscr{D}$ and for some $\beta>0$, clearly, Theorem 5.1 applies with $\psi_{d, x}=d(x, \cdot)^{\beta}$. Under these last assumptions, and if, in addition, the subspace $E$ verifies the integral representation property $(\mathrm{P})$, then Theorem 5.1 is an obvious consequence of Theorems 3.1, 4.4, and 4.5.

If, in addition, $X$ is bounded, then, according to Remark 3.2(4), Theorem 5.1, in turn, implies Theorems 3.1, 4.4, and 4.5.

(3) If $X$ is a metric space, then results similar to Theorem 5.1 which involve other particular classes of test functions can also be found in [19].

Now we will briefly discuss a simple application of Theorem 5.1.

Let $X$ be a set and consider a subset $S$ of $\mathscr{F}(X, \mathbb{R})$ which separates the points of $X$. For every $m \geq 1$ and for every $\varphi_{1}, \ldots, \varphi_{m} \in S$, consider the pseudometric $d_{\varphi_{1}, \ldots, \varphi_{m}}: X \times X \rightarrow \mathbb{R}$ defined by

$$
d_{\varphi_{1}, \ldots, \varphi_{m}}(x, y):=\sup _{1 \leq i \leq m}\left|\varphi_{i}(x)-\varphi_{i}(y)\right| \quad(x, y \in X)
$$

The family

$$
\mathscr{D}:=\left\{d_{\varphi_{1}, \ldots, \varphi_{m}} \mid m \geq 1, \varphi_{1}, \ldots, \varphi_{m} \in S\right\}
$$

is saturated and generates a topology $\mathscr{T}_{S}$ on $X$ with respect to which $X$ is a completely regular Hausdorff space. The topology $\mathscr{T}_{S}$ is the coarsest topology of $X$ with respect to which each function $\varphi \in S$ is continuous.

Thus, if $(X, \mathscr{T})$ is a topological space and $S \subset \mathscr{C}(X, \mathbb{R})$, then $\mathscr{T}_{S} \subset \mathcal{T}$. If $(X, \mathscr{T})$ is a compact Hausdorff space, then $\mathscr{T}_{S}=\mathscr{T}$. If $(X, \mathscr{T})$ is a locally compact Hausdorff space, $S$ is a subset of continuous functions on $X$ which are convergent to the point at infinity $\infty$ of $X$ and which separate the points of $X_{\infty}$, that is, $S$ separates the points of $X$, and for any $x \in X$, there exists $\varphi \in S$ such that $\varphi(x) \neq \lim _{y \rightarrow \infty} \varphi(y)$, then again $\mathscr{T}_{S}=\mathscr{T}$.

Consider, indeed, the one-point compactification $\left(X_{\infty}, \mathcal{T}_{\infty}\right)$ of $X$, where $X_{\infty}:=$ $X \cup\{\infty\}$ (see [17, Subsection 3.15, page 45]), and for any $\varphi \in S$, denote by $\tilde{\varphi}: X_{\infty} \rightarrow \mathbb{R}$ the function defined by

$$
\tilde{\varphi}(x):= \begin{cases}\varphi(x), & x \in X, \\ \lim _{x \rightarrow \infty} \varphi(x), & x=\infty .\end{cases}
$$

Then the subset $\tilde{S}:=\{\tilde{\varphi}: \varphi \in S\}$ is contained in $\mathscr{C}\left(X_{\infty}, \mathbb{R}\right)$ and separates the points of $X_{\infty}$, and hence $\mathscr{T}_{\tilde{S}}=\mathscr{T}_{\infty}$.

On the other hand, since $\widetilde{\varphi}_{\mid X}=\varphi$ for every $\varphi \in S$, then (see $[17$, Subsection 15.8 , page 221])

$$
\mathscr{T}_{S}=\mathscr{T}_{\tilde{S} \mid X}=\mathscr{T}_{\infty \mid X}=\mathscr{T} .
$$


From now on, we will fix a set $X$ and a subset $S$ of $\mathscr{F}(X, \mathbb{R})$ which separates the points of $X$. On $X$ we will consider the topology $\mathscr{T}_{S}$ generated by the family of pseudometrics (5.17).

The spaces of all real-valued functions on $X$ which are continuous (resp., bounded and continuous, bounded and uniformly continuous) with respect to $\mathscr{T}_{S}$ will be denoted by $\mathscr{C}_{S}(X, \mathbb{R}), \mathscr{C}_{S, b}(X, \mathbb{R})$ and $U \mathscr{C}_{S, b}(X, \mathbb{R})$. The symbol $\operatorname{Lip}_{S}(X, \alpha)$ denotes the corresponding space of Hölder continuous functions with exponent $\alpha>0$.

THEOREM 5.3. Let $E$ be a vector subspace of $\mathscr{F}(X, \mathbb{R})$ such that $\{\mathbf{1}\} \cup S \cup S^{2} \subset$ $E$, where $S^{2}:=\left\{\varphi^{2} \mid \varphi \in S\right\}$. Let $\left(L_{i}\right)_{i \in I}$ be a net of positive linear operators from $E$ into $\mathscr{F}(X, \mathbb{R})$ such that $\lim _{i \in I}^{\leq} L_{i}(\varphi)=\varphi$ uniformly on $X$ (resp., pointwise on $X$ ) for every $\varphi \in\{\mathbf{1}\} \cup S \cup S^{2}$.

Then,

$$
\lim _{i \in I}^{\leq} L_{i}(f)=f
$$

uniformly on $X$ for every $f \in E \cap U C_{S, b}(X, \mathbb{R})$, respectively,

$$
\lim _{i \in I} \leq L_{i}(f)=f
$$

pointwise on $X$ for every $f \in E \cap \mathscr{C}_{S, b}(X, \mathbb{R})$. Moreover, if $\left(X, \mathscr{T}_{S}\right)$ is locally compact,

$$
\lim _{i \in I}^{\leq} L_{i}(f)=f
$$

uniformly on compact subsets of $X$ for every $f \in E \cap \mathscr{C}_{s, b}(X, \mathbb{R})$.

Finally, the limits (5.20) and (5.22) (resp., the limit (5.21)) hold true uniformly with respect to $f \in H$ provided $H$ is an equibounded and uniformly equicontinuous (resp., equicontinuous) subset of $E \cap \mathscr{C}_{S, b}(X, \mathbb{R})$.

PRoof. For every $d=d_{\varphi_{1}, \ldots, \varphi_{m}} \in \mathscr{D}$ and for every $x \in X$, set

$$
\psi_{d, x}:=\sum_{h=1}^{m}\left(\varphi_{h}(x)-\varphi_{h}\right)^{2} \in E .
$$

Then $d(x, \cdot)^{2} \leq \psi_{d, x}$ and

$$
\lim _{i \in I} \leq L_{i}\left(\psi_{x, d}\right)(x)=\lim _{i \in I} \leq \sum_{h=1}^{m} L_{i}\left(\left(\varphi_{h}(x)-\varphi_{h}\right)^{2}\right)(x)=0
$$

uniformly with respect to $x \in X$ (resp., pointwise on $X$ ).

The result now follows from Theorem 5.1.

In order to provide some estimates of the rates of the convergence considered in Theorem 5.3, it is useful to introduce the following quantities which 
will replace the corresponding ones defined by (3.17) and (3.18), respectively, by considering the system of pseudometrics (5.17).

Given $\varphi_{1}, \ldots, \varphi_{m} \in S, m \geq 1$, for any $f \in \mathscr{F}(X, \mathbb{R}), \delta>0$, and $x \in X$, we set

$$
\begin{aligned}
& \widetilde{\omega}_{\varphi_{1}, \ldots, \varphi_{m} ; x}(f, \delta) \\
& \quad:=\sup \left\{|f(x)-f(y)|: y \in X, \sum_{h=1}^{n}\left(\varphi_{h}(x)-\varphi_{h}(y)\right)^{2} \leq \delta^{2}\right\}, \\
& \widetilde{\omega}_{\varphi_{1}, \ldots, \varphi_{m}}(f, \delta) \\
& \quad:=\sup \left\{|f(x)-f(y)|: x, y \in X, \sum_{h=1}^{n}\left(\varphi_{h}(x)-\varphi_{h}(y)\right)^{2} \leq \delta^{2}\right\} ;
\end{aligned}
$$

both $\widetilde{\omega}_{\varphi_{1}, \ldots, \varphi_{m} ; x}(f, \delta)$ and $\widetilde{\omega}_{\varphi_{1}, \ldots, \varphi_{m}}(f, \delta)$ belong to $\mathbb{R} \cup\{+\infty\}$.

We have the following result.

Proposition 5.4. Under the same assumptions of Theorem 5.3, for every $f \in E \cap \mathscr{C}_{s, b}(X, \mathbb{R}), i \in I, \delta>0, \varphi_{1}, \ldots, \varphi_{m} \in S, m \geq 1$, and $x \in X$,

$$
\begin{aligned}
\left|L_{i} f(x)-f(x)\right| \leq & \|f\|_{\infty}\left|L_{i}(\mathbf{1})(x)-1\right|+\widetilde{\omega}_{\varphi_{1}, \ldots, \varphi_{m} ; x}(f, \delta) L_{i}(\mathbf{1})(x) \\
& +\frac{2\|f\|_{\infty}}{\delta^{2}} \sum_{h=1}^{m} L_{i}\left(\left(\varphi_{h}(x)-\varphi_{h}\right)^{2}\right)(x) .
\end{aligned}
$$

If $f \in E \cap U C_{S, b}(X, \mathbb{R})$,

$$
\begin{aligned}
\left\|L_{i}(f)-f\right\|_{\infty} \leq & \|f\|_{\infty}\left\|L_{i}(\mathbf{1})-\mathbf{1}\right\|_{\infty}+\widetilde{\omega}_{\varphi_{1}, \ldots, \varphi_{m}}(f, \delta)\left\|L_{i}(\mathbf{1})\right\|_{\infty} \\
& +\frac{2\|f\|_{\infty}}{\delta^{2}} \sup _{x \in X} \sum_{h=1}^{m} L_{i}\left(\left(\varphi_{h}(x)-\varphi_{h}\right)^{2}\right)(x) .
\end{aligned}
$$

Finally, if $f \in E \cap \mathscr{C}_{S, b}(X, \mathbb{R}) \cap \operatorname{Lip}_{S}(X, \alpha)$, with $0<\alpha \leq 2$, then there exist $M \geq 0$ and $\varphi_{1}, \ldots, \varphi_{m} \in S$ such that for every $i \in I$ and $\delta>0$,

$$
\begin{aligned}
\left\|L_{i}(f)-f\right\|_{\infty} \leq & \|f\|_{\infty}\left\|L_{i}(\mathbf{1})-\mathbf{1}\right\|_{\infty}+M \delta^{\alpha}\left\|L_{i}(\mathbf{1})\right\|_{\infty} \\
& +\frac{M}{\delta^{2-\alpha}} \sup _{x \in X} \sum_{h=1}^{m} L_{i}\left(\left(\varphi_{h}(x)-\varphi_{h}\right)^{2}\right)(x)
\end{aligned}
$$

and, in particular,

$$
\begin{aligned}
\left\|L_{i}(f)-f\right\|_{\infty} \leq & \|f\|_{\infty}\left\|L_{i}(\mathbf{1})-\mathbf{1}\right\|_{\infty}+M\left(\left\|L_{i}(\mathbf{1})\right\|_{\infty}+1\right) \\
& \times\left(\sup _{x \in X} \sum_{h=1}^{m} L_{i}\left(\left(\varphi_{h}(x)-\varphi_{h}\right)^{2}\right)(x)\right)^{\alpha / 2} .
\end{aligned}
$$

Proof. Since for every $x, y \in X$, we have

$$
|f(x)-f(y)| \leq \widetilde{\omega}_{\varphi_{1}, \ldots, \varphi_{m} ; x}(f, \delta)+\frac{2\|f(x)\|_{\infty}}{\delta^{2}} \sum_{h=1}^{m}\left(\varphi_{h}(x)-\varphi_{h}(y)\right)^{2},
$$


we get

$$
\begin{aligned}
\left|L_{i}(f)-f(x) L_{i}(\mathbf{1})\right| \leq & \widetilde{\omega}_{\varphi_{1}, \ldots, \varphi_{m} ; x}(f, \delta) L_{i}(\mathbf{1}) \\
& +\frac{2\|f\|_{\infty}}{\delta^{2}} \sum_{h=1}^{m} L_{i}\left(\left(\varphi_{h}(x)-\varphi_{h}\right)\right),
\end{aligned}
$$

and hence (5.27) and (5.28) follow.

The estimates (5.29) and (5.30) directly come from (5.13) and (5.14).

REMARK 5.5. (1) Theorem 5.3 extends and generalizes several useful Korovkin-type theorems which have been previously established in the setting of compact spaces (see [1, Section 4.4 and the final Notes and references]).

(2) The idea of associating a metric such as (5.16) to finitely many test functions has already been used in [19] when $X$ is a subset of some Euclidean space (see also [23], where an abstract modulus of continuity similar to (5.26) is also introduced in the case when $X$ is a convex compact subset of a locally convex space).

6. Some positive approximation processes on convex subsets. In this section, we will present some applications of the previous results in the setting of spaces of weakly continuous functions defined on a convex subset of a locally convex space.

Let $Y$ be a locally convex Hausdorff space and let $Y^{\prime}$ be its topological dual. Consider a convex subset $X$ of $Y$, and denote by

$$
Y_{X}^{\prime}:=\left\{\varphi_{\mid X}: \varphi \in Y^{\prime}\right\}
$$

the set of all the restrictions to $X$ of the continuous linear functionals on $Y$.

In this case, the topology $\mathscr{T}_{Y_{X}^{\prime}}$ introduced in Section 5 coincides with the weak topology $\mathscr{T}_{w}$ on $X$. Thus, in the sequel, we will use the symbols $\mathfrak{b}_{w, b}(X, \mathbb{R})$ and $U C_{w, b}(X, \mathbb{R})$ to denote the spaces of all real-bounded functions on $X$ which are weakly continuous and, respectively, uniformly weakly continuous.

Moreover, the symbol $\mathcal{M}_{w}^{+}(X)$ (resp., $\mathcal{M}_{b, w}^{+}(X)$ and $\left.\mathcal{M}_{1, w}^{+}(X)\right)$ will denote the cone of all positive (resp., positive and bounded, probability) measures defined on the Borel $\sigma$-algebra generated by $\mathscr{T}_{w}$ on $X$ (which is included in the natural Borel $\sigma$-algebra of $X$ ).

We recall that the topology $\mathscr{T}_{w}$ is separated as a consequence of the HahnBanach theorem.

From now on, we will fix a nontrivial family $\left(\mu_{x}\right)_{x \in X}$ in $\mu_{1, w}^{+}(X)$ such that, for every $x \in X$ and $\varphi \in Y^{\prime}$,

$$
\begin{gathered}
\left.\varphi\right|_{X} \in \bigcap_{x \in X} \mathscr{L}^{1}\left(X, \mu_{x}\right), \\
\left.\int_{X} \varphi\right|_{X} d \mu_{x}=\varphi(x) .
\end{gathered}
$$


Here the term "nontrivial" means that $\mu_{x} \neq \varepsilon_{x}$, with $\varepsilon_{x}$ being the point mass at $x$, for every $x \in X$.

Notice that, since the measures $\mu_{x}$ are bounded, the following inclusion holds true:

$$
\mathscr{C}_{w, b}(X, \mathbb{R}) \subset \bigcap_{x \in X} \mathscr{L}^{1}\left(X, \mu_{x}\right)
$$

Moreover, from Hölder's inequality and from (6.3), it also follows that

$$
\varphi^{2}(x) \leq \int_{X} \varphi_{\mid X}^{2} d \mu_{x} \quad\left(\varphi \in Y^{\prime}, x \in X\right) .
$$

For every $n \geq 1$, consider the mapping $\pi_{n}: X^{n} \rightarrow X$ defined by

$$
\pi_{n}\left(x_{1}, \ldots, x_{n}\right):=\frac{x_{1}+\cdots+x_{n}}{n} \quad\left(x_{1}, \ldots, x_{n} \in X\right)
$$

and denote by

$$
\mu_{x, n}:=\pi_{n}\left(\mu_{x} \otimes \cdots \otimes \mu_{x}\right) \quad(x \in X, n \geq 1)
$$

the image measure of the $n$-times tensorial product $\mu_{x} \otimes \cdots \otimes \mu_{x}$ under $\pi_{n}$.

In this case, the sequence of positive linear operators associated with (6.7) is given by

$$
B_{n}(f)(x)=\int_{X} \cdots \int_{X} f\left(\frac{x_{1}+\cdots+x_{n}}{n}\right) d \mu_{x}\left(x_{1}\right) \cdots d \mu_{x}\left(x_{n}\right)
$$

for every $n \geq 1, x \in X$, and $f \in E:=\bigcap_{x \in X, n \geq 1} \mathscr{L}^{1}\left(X, \mu_{x, n}\right)$. These operators have been extensively studied for compact convex sets (see [1, Chapter 6] for details) and are called Bernstein-Schnabl operators.

More generally, for every $n \geq 1$, consider a mapping $\sigma_{n}: X \rightarrow X$ and set

$$
\eta_{x, n}:=\pi_{n}\left(\bigotimes_{h=1}^{n} \mu_{\sigma_{h}(x)}\right) \quad(n \geq 1, x \in X) .
$$

In this case, the operators associated with (6.9) are defined as

$$
A_{n}(f)(x)=\int_{X} \cdots \int_{X} f\left(\frac{x_{1}+\cdots+x_{n}}{n}\right) d \mu_{\sigma_{1}(x)}\left(x_{1}\right) \cdots d \mu_{\sigma_{n}(x)}\left(x_{n}\right)
$$

for every $n \geq 1, x \in X$, and $f \in E:=\bigcap_{x \in X, n \geq 1} \mathscr{L}^{1}\left(X, \eta_{x, n}\right)$.

Notice that if the mapping $X \mapsto \mu_{X}$ is continuous with respect to the weak topology on $X$ and the weak topology on $\mu_{1, w}^{+}(X)$-that is, for every $f \in$ $\mathscr{C}_{w, b}(X, \mathbb{R})$, the function $x \mapsto \int_{X} f d \mu_{x}$ is weakly continuous-then there $B_{n}(f)$ $\in \mathscr{C}_{w, b}(X, \mathbb{R})\left(f \in \mathscr{C}_{w, b}(X, \mathbb{R}), n \geq 1\right)$.

If, in addition, each mapping $\sigma_{n}$ is weak-to-weak continuous, then $A_{n}(f) \in$ $\mathscr{C}_{w, b}(X, \mathbb{R})\left(f \in \mathscr{C}_{w, b}(X, \mathbb{R}), n \geq 1\right)$. 
EXAMPLE 6.1. (1) Set $X:=[0,1]$ and consider the family $\left(\mu_{x}\right)_{0 \leq x \leq 1}$ defined by

$$
\mu_{x}:=(1-x) \varepsilon_{0}+x \varepsilon_{1} \quad(0 \leq x \leq 1)
$$

then the operators defined by (6.8) are the Bernstein operators, while the sequence (6.10) becomes

$$
A_{n}(f)(x)=\sum_{h=0}^{n} b_{h}(x) f\left(\frac{h}{n}\right)
$$

for every $f \in \mathscr{F}_{(}([0,1], \mathbb{R})$, where for $h=0,1, \ldots, n$,

$$
\begin{aligned}
& b_{h}(x) \\
&:= \begin{cases}\prod_{i=1}^{n}\left(1-\sigma_{i}(x)\right), & h=0, \\
\sum_{1 \leq i_{1}<\cdots<i_{h} \leq n} \prod_{\substack{1 \leq i \leq n \\
i \neq i_{1}, \ldots, i_{h}}}\left(1-\sigma_{i}(x)\right) \prod_{i=i_{1}, \ldots, i_{h}} \sigma_{i}(x), & 1 \leq h \leq n-1, \\
\prod_{i=1}^{n} \sigma_{i}(x), & h=n .\end{cases}
\end{aligned}
$$

Moreover, the operators in (6.12) were first introduced by King [20] by a completely different method (see also [1, Subsection 5.2.5, page 294]).

(2) Let $X:=\left[0,+\infty\left[\right.\right.$ and consider the family $\left(\mu_{x}\right)_{x \geq 0}$ defined by

$$
\mu_{x}:=\sum_{h=0}^{+\infty} e^{-x} \frac{x^{h}}{h !} \varepsilon_{h} \quad(x \geq 0)
$$

In this case, we get

$$
A_{n}(f)(x)=\sum_{h=0}^{\infty} e^{-\left(\sigma_{1}(x)+\cdots+\sigma_{n}(x)\right)} \frac{\left(\sigma_{1}(x)+\cdots+\sigma_{n}(x)\right)^{h}}{h !} f\left(\frac{h}{n}\right)
$$

for every $n \geq 1, x \geq 0$, and $f \in E:=\bigcap_{x \geq 0, n \geq 1} \mathscr{L}^{1}\left(\left[0,+\infty\left[, \eta_{x, n}\right)\right.\right.$, while the operators $B_{n}$ defined by (6.8) are the Favard-Szász-Mirakjan operators.

(3) Finally, for $X:=\mathbb{R}$, set for every $x \in \mathbb{R}$,

$$
\mu_{x}:=g_{x, 1} \lambda_{1}
$$

that is, $\mu_{x}$ denotes the measure with density $g_{x, 1}$ relative to the Lebesgue measure on $\mathbb{R}$, where $g_{x, 1}$ is the normal density defined by

$$
g_{x, 1}(t):=\frac{1}{\sqrt{2 \pi}} e^{-(1 / 2)(t-x)^{2}} \quad(t \in \mathbb{R}) .
$$


In this case, using some properties of the normal densities, we get

$$
\eta_{x, n}=g_{\left(\sigma_{1}(x)+\cdots+\sigma_{n}(x)\right) / n, 1 / n} \cdot \lambda_{1} \quad(x \in \mathbb{R}, n \geq 1),
$$

where

$$
g_{\left(\sigma_{1}(x)+\cdots+\sigma_{n}(x)\right) / n, 1 / n}(t)=\sqrt{\frac{n}{2 \pi}} e^{-(n / 2)\left(t-\left(\sigma_{1}(x)+\cdots+\sigma_{n}(x)\right) / n\right)^{2}} \quad(t \in \mathbb{R}),
$$

and hence

$$
A_{n}(f)(x)=\sqrt{\frac{n}{2 \pi}} \int_{-\infty}^{+\infty} f(t) e^{-(n / 2)\left(t-\left(\sigma_{1}(x)+\cdots+\sigma_{n}(x)\right) / n\right)^{2}} d t
$$

for every $n \geq 1, x \in \mathbb{R}$, and $f \in E:=\bigcap_{x \in \mathbb{R}, n \geq 1} \mathscr{L}^{1}\left(\mathbb{R}, \eta_{x, n}\right)$.

In a similar way, one can show that the operators $B_{n}$ defined by (6.8) are given by

$$
B_{n}(f)(x)=\sqrt{\frac{n}{2 \pi}} \int_{-\infty}^{+\infty} f(t) e^{-(n / 2)(t-x)^{2}} d t
$$

(Gauss-Weierstrass operators).

From Theorem 5.3, we may derive the following result concerning the approximation properties of the operators (6.8) and (6.10).

We will keep the same notation so far introduced.

THEOREM 6.2. Assume that for every $\varphi \in Y_{X}^{\prime}$,

$$
\varphi^{2} \in \bigcap_{x \in X} \mathscr{L}^{1}\left(X, \mu_{x}\right)
$$

Then the following statements hold true.

(1) For every $f \in \mathscr{C}_{w, b}(X, \mathbb{R}), \lim _{n \rightarrow \infty} B_{n}(f)=f$ pointwise on $X$. If, in addition,

$$
\sup _{x \in X}\left(\int_{X} \varphi^{2} d \mu_{x}-\varphi^{2}(x)\right)<+\infty \quad \forall \varphi \in Y_{X}^{\prime}
$$

then $\lim _{n \rightarrow \infty} B_{n}(f)=f$ uniformly on $X$ for every $f \in U C_{w, b}(X, \mathbb{R})$.

Moreover, if $\left(X, \mathcal{T}_{w}\right)$ is locally compact, then $\lim _{n \rightarrow \infty} B_{n}(f)=f$ uniformly on compact subsets of $X$ for every $f \in \mathscr{C}_{w, b}(X, \mathbb{R})$.

(2) If for every $x \in X$,

$$
\lim _{n \rightarrow+\infty} \frac{1}{n} \sum_{i=1}^{n} \sigma_{i}(x)=x \quad \text { weakly on } X,
$$

that is, for any $x \in X$,

$$
\lim _{n \rightarrow+\infty} \varphi\left(\frac{\sigma_{1}(x)+\cdots+\sigma_{n}(x)}{n}\right)=\varphi(x) \quad \forall \varphi \in Y_{X}^{\prime},
$$


(resp., (6.24) holds uniformly with respect to $x \in X)$, and if

$$
\lim _{n \rightarrow+\infty} \frac{1}{n^{2}} \sum_{i=1}^{n}\left(\int_{X} \varphi^{2} d \mu_{\sigma_{i}(x)}-\varphi^{2}\left(\sigma_{i}(x)\right)\right)=0 \quad \forall \varphi \in Y_{X}^{\prime},
$$

(resp., $\left.\lim _{n \rightarrow+\infty}\left(1 / n^{2}\right) \sup _{x \in X} \sum_{i=1}^{n}\left(\int_{X} \varphi^{2} d \mu_{\sigma_{i}(x)}-\varphi^{2}\left(\sigma_{i}(x)\right)\right)=0\right)$, then for every $f \in \mathscr{C}_{w, b}(X, \mathbb{R}), \lim _{n \rightarrow+\infty} A_{n}(f)=f$ pointwise on $X$ (resp., for every $f \in$ $U C_{w, b}(X, \mathbb{R}), \lim _{n \rightarrow+\infty} A_{n}(f)=f$ uniformly on $\left.X\right)$.

Moreover, if $\left(X, \mathcal{T}_{w}\right)$ is locally compact, then $\lim _{n \rightarrow \infty} A_{n}(f)=f$ uniformly on compact subsets of $X$ for every $f \in \mathscr{C}_{w, b}(X, \mathbb{R})$.

Proof. (1) First notice that for each $x \in X, n \geq 1$, and $\varphi \in Y_{X}^{\prime}$, we have

$$
\begin{aligned}
B_{n}(\mathbf{1})(x) & :=\mu_{x, n}(X)=1, \\
B_{n}(\varphi)(x) & :=\int_{X} \varphi d \mu_{x, n}=\varphi(x), \\
B_{n}\left(\varphi^{2}\right)(x) & :=\int_{X} \varphi^{2} d \mu_{x, n}=\varphi^{2}(x)+\frac{1}{n}\left(\int_{X} \varphi^{2} d \mu_{x}-\varphi^{2}(x)\right) .
\end{aligned}
$$

Hence, for any $\varphi \in\{\mathbf{1}\} \cup Y_{X}^{\prime} \cup\left(Y_{X}^{\prime}\right)^{2}, B_{n}(\varphi) \rightarrow \varphi$ pointwise on $X$, therefore Theorem 5.3 applies.

With the same reasoning, but taking (6.23) into account, we get the uniform convergence on $X$. The last part of assertion (1) comes directly from Theorem 5.3.

(2) We intend to apply Theorem 5.3 again to the sequence $\left(A_{n}\right)_{n \geq 1}$.

To this aim, observe that

$$
\begin{aligned}
A_{n}(\mathbf{1})(x):= & \eta_{x, n}(X)=1, \\
A_{n}(\varphi)(x):= & \int_{X} \varphi d \eta_{x, n}=\varphi\left(\frac{\sigma_{1}(x)+\cdots+\sigma_{n}(x)}{n}\right), \\
A_{n}\left(\varphi^{2}\right)(x):= & \int_{X} \varphi^{2} d \eta_{x, n}=\frac{1}{n^{2}} \sum_{i=1}^{n}\left(\int_{X} \varphi^{2} d \mu_{\sigma_{i}(x)}-\varphi^{2}\left(\sigma_{i}(x)\right)\right) \\
& +\varphi^{2}\left(\frac{\sigma_{1}(x)+\cdots+\sigma_{n}(x)}{n}\right)
\end{aligned}
$$

for every $n \geq 1, x \in X$, and $\varphi \in Y_{X}^{\prime}$.

Then, by virtue of (6.24) and (6.26), $A_{n}(\varphi) \rightarrow \varphi$ pointwise on $X$ for any $\varphi \in\{\mathbf{1}\} \cup Y_{X}^{\prime} \cup\left(Y_{X}^{\prime}\right)^{2}$, and therefore Theorem 5.3 applies.

The uniform case is analogous.

REMARK 6.3. We examine the behavior of the sequences of operators introduced in Example 6.1 on the light of Theorem 6.2. 
First notice that, in all the three cases, $Y_{X}^{\prime}=\left\{e_{1}\right\}$, where $e_{1}(x):=x(x \in X)$. Moreover, condition (6.24) is equivalent to

$$
\lim _{n \rightarrow+\infty} \frac{1}{n} \sum_{i=1}^{n} \sigma_{i}(x)=x \quad(x \in X) .
$$

For every $x \in X$, set $e_{2}(x):=x^{2}=e_{1}(x)^{2}$.

(1) First consider the family (6.11) and observe that

$$
\int_{X} e_{2} d \mu_{\sigma_{n}(x)}-e_{2}\left(\sigma_{n}(x)\right)=\sigma_{n}(x)\left(1-\sigma_{n}(x)\right)
$$

so that (6.26) holds uniformly on $[0,1]$.

Therefore, if (6.29) holds true uniformly on $[0,1]$, then for every $f \in \mathscr{C}([0,1])$, we have that $A_{n}(f) \rightarrow f$ uniformly on $[0,1]$, where the operators $A_{n}$ are defined by (6.12). This shows, in particular, that if the functions $\sigma_{n}$ are continuous, the subalgebra generated by $\{\mathbf{1}\} \cup\left\{\sigma_{n}: n \geq 1\right\}$ is dense in $\mathscr{b}([0,1])$, and the sequence $\left(A_{n}(f)\right)_{n \geq 1}$ represents an explicit example of a sequence of elements of such a subalgebra which converges uniformly to $f$.

(2) Now consider the measures (6.14). In this case,

$$
\int_{X} e_{2} d \mu_{\sigma_{n}(x)}-e_{2}\left(\sigma_{n}(x)\right)=\sigma_{n}(x),
$$

thus (6.26) becomes

$$
\lim _{n \rightarrow+\infty} \frac{\sigma_{n}(x)}{n^{2}}=0
$$

which is satisfied pointwise, but in general not uniformly on $[0,+\infty[$.

(3) Finally, take the measures (6.16) into account. Since

$$
\int_{X} e_{2} d \mu_{\sigma_{n}(x)}-e_{2}\left(\sigma_{n}(x)\right)=1,
$$

(6.26) is uniformly satisfied on $\mathbb{R}$ and (6.23) holds too.

Our next purpose is to evaluate the rates of convergence analyzed in Theorem 6.2. To this aim we remark that, in this case, the moduli (5.25) and (5.26) become

$$
\begin{aligned}
\omega_{x}\left(f ; \varphi_{1}, \ldots, \varphi_{m}, \delta\right) & :=\sup \left\{|f(x)-f(y)|: y \in X, \sum_{h=1}^{n} \varphi_{h}^{2}(x-y) \leq \delta^{2}\right\}, \\
\omega\left(f ; \varphi_{1}, \ldots, \varphi_{m}, \delta\right) & :=\sup \left\{|f(x)-f(y)|: x, y \in X, \sum_{h=1}^{n} \varphi_{h}^{2}(x-y) \leq \delta^{2}\right\},
\end{aligned}
$$

for all $f \in \mathscr{C}_{w, b}(X, \mathbb{R}), \delta>0, \varphi_{1}, \ldots, \varphi_{m} \in Y_{X}^{\prime}, m \geq 1$, and $x \in X$. 
These moduli of continuity have been already considered in [1, Section 5.1, page 270] in the setting of a convex compact subset of a locally convex space. However, they were first introduced and studied by Nishishiraho [23, 24, 25].

Proposition 6.4. Under the assumption of Theorem 6.2, the following statements hold true.

(1) For any $n, m \geq 1, x \in X, \delta>0$, and $\varphi_{1}, \ldots, \varphi_{m} \in Y_{X}^{\prime}$, if $f \in \mathscr{C}_{w, b}(X, \mathbb{R})$, then

$$
\begin{aligned}
\left|B_{n}(f)(x)-f(x)\right| \leq & \omega_{x}\left(f ; \varphi_{1}, \ldots, \varphi_{m}, \delta\right) \\
& +2 \frac{\|f\|_{\infty}}{n \delta^{2}} \sum_{h=1}^{m}\left(\int_{X} \varphi_{h}^{2} d \mu_{x}-\varphi_{h}^{2}(x)\right),
\end{aligned}
$$

and if $f \in U C_{w, b}(X, \mathbb{R})$,

$$
\begin{aligned}
\left\|B_{n}(f)-f\right\|_{\infty} \leq & \omega\left(f ; \varphi_{1}, \ldots, \varphi_{m}, \delta\right) \\
& +2 \frac{\|f\|_{\infty}}{n \delta^{2}} \sup _{x \in X} \sum_{h=1}^{m}\left(\int_{X} \varphi_{h}^{2} d \mu_{x}-\varphi_{h}^{2}(x)\right) .
\end{aligned}
$$

Moreover, if $f \in \mathscr{C}_{w, b}(X, \mathbb{R}) \cap \operatorname{Lip}(X, \alpha)$, with $0<\alpha \leq 2$, then there exist $M \geq 0$ and $\varphi_{1}, \ldots, \varphi_{m} \in Y_{X}^{\prime}(m \geq 1)$ so that for any $\delta>0$,

$$
\left\|B_{n}(f)-f\right\|_{\infty} \leq M \delta^{\alpha}+\frac{M \delta^{\alpha-2}}{n} \sup _{x \in X} \sum_{h=1}^{m}\left(\int_{X} \varphi_{h}^{2} d \mu_{x}-\varphi_{h}^{2}(x)\right) .
$$

(2) For any $n, m \geq 1, x \in X, \delta>0$, and $\varphi_{1}, \ldots, \varphi_{m} \in Y_{X}^{\prime}$, if $f \in \mathscr{C}_{w, b}(X, \mathbb{R})$, then

$$
\begin{aligned}
& \left|A_{n}(f)(x)-f(x)\right| \\
& \leq \omega_{x}\left(f ; \varphi_{1}, \ldots, \varphi_{m}, \delta\right) \\
& +2 \frac{\|f\|_{\infty}}{\delta^{2}} \sum_{h=1}^{m}\left[\varphi_{h}^{2}(x)-2 \varphi_{h}(x) \varphi_{h}\left(\frac{1}{n} \sum_{i=1}^{n} \sigma_{i}(x)\right)\right. \\
& +\varphi_{h}^{2}\left(\frac{1}{n} \sum_{i=1}^{n} \sigma_{i}(x)\right) \\
& \left.\quad+\frac{1}{n^{2}} \sum_{i=1}^{n}\left(\int_{X} \varphi_{h}^{2} d \mu_{\sigma_{i}(x)}-\varphi_{h}^{2}\left(\sigma_{i}(x)\right)\right)\right],
\end{aligned}
$$


and if $f \in U C_{w, b}(X, \mathbb{R})$,

$$
\begin{aligned}
&\left\|A_{n}(f)-f\right\|_{\infty} \\
& \leq \omega\left(f ; \varphi_{1}, \ldots, \varphi_{m}, \delta\right) \\
&+2 \frac{\|f\|_{\infty}}{\delta^{2}} \sup _{x \in X} \sum_{h=1}^{m}\left[\varphi_{h}^{2}(x)-2 \varphi_{h}(x) \varphi_{h}\left(\frac{1}{n} \sum_{i=1}^{n} \sigma_{i}(x)\right)\right. \\
&+\varphi_{h}^{2}\left(\frac{1}{n} \sum_{i=1}^{n} \sigma_{i}(x)\right) \\
&\left.+\frac{1}{n^{2}} \sum_{i=1}^{n}\left(\int_{X} \varphi_{h}^{2} d \mu_{\sigma_{i}(x)}-\varphi_{h}^{2}\left(\sigma_{i}(x)\right)\right)\right] .
\end{aligned}
$$

Finally, if $f \in \mathscr{C}_{w, b}(X, \mathbb{R}) \cap \operatorname{Lip}(X, \alpha)$, with $0<\alpha \leq 2$, then there exist $M \geq 0$ and $\varphi_{1}, \ldots, \varphi_{m} \in Y_{X}^{\prime}(m \geq 1)$ so that for any $\delta>0$,

$$
\begin{aligned}
\left\|A_{n}(f)-f\right\|_{\infty} \leq & M \delta^{\alpha} \\
+M \delta^{\alpha-2} \sup _{x \in X} \sum_{h=1}^{m}\left[\varphi_{h}^{2}(x)-\varphi_{h}(x) \varphi_{h}\left(\frac{1}{n} \sum_{i=1}^{n} \sigma_{i}(x)\right)\right. & \\
& +\varphi_{h}^{2}\left(\frac{1}{n} \sum_{i=1}^{n} \sigma_{i}(x)\right) \\
& \left.+\frac{1}{n^{2}} \sum_{i=1}^{n}\left(\int_{X} \varphi_{h}^{2} d \mu_{\sigma_{i}(x)}-\varphi_{h}^{2}\left(\sigma_{i}(x)\right)\right)\right] .
\end{aligned}
$$

Proof. It suffices to apply the general results obtained in Proposition 5.4 to the operators $A_{n}$ and $B_{n}$, taking the linearity of the functionals $\varphi_{1}, \ldots, \varphi_{m} \in Y_{X}^{\prime}$ and formulas (6.27) and (6.28) into account.

ACKNOWLEDgment. We thank the anonymous referee for his comments.

\section{REFERENCES}

[1] F. Altomare and M. Campiti, Korovkin-Type Approximation Theory and Its Applications, de Gruyter Studies in Mathematics, vol. 17, Walter de Gruyter, Berlin, 1994.

[2] F. Altomare and I. Carbone, On a new sequence of positive linear operators on unbounded intervals, Rend. Circ. Mat. Palermo (2) Suppl. (1996), no. 40, 23-36.

[3] F. Altomare and E. M. Mangino, On a generalization of Baskakov operators, Rev. Roumaine Math. Pures Appl. 44 (1999), no. 5-6, 683-705.

[4] F. Altomare and I. Raşa, Towards a characterization of a class of differential operators associated with positive projections, Atti Sem. Mat. Fis. Univ. Modena 46 (1998), no. suppl., 3-38. 
[5] D. Andrica and C. Mustăţa, An abstract Korovkin type theorem and applications, Studia Univ. Babeş-Bolyai Math. 34 (1989), no. 2, 44-51.

[6] H. Bauer, Probability Theory, de Gruyter Studies in Mathematics, vol. 23, Walter de Gruyter, Berlin, 1996.

[7] _ Measure and Integration Theory, de Gruyter Studies in Mathematics, vol. 26, Walter de Gruyter, Berlin, 2001.

[8] C. Berg, J. P. R. Christensen, and P. Ressel, Harmonic Analysis on Semigroups, Graduate Texts in Mathematics, vol. 100, Springer-Verlag, New York, 1984.

[9] G. Choquet, Lectures on Analysis. Vol. I: Integration and Topological Vector Spaces, W. A. Benjamin, New York, 1969.

[10] _ Lectures on Analysis. Vol. II: Representation Theory, W. A. Benjamin, New York, 1969.

[11] J. de la Cal and F. Luquin, Probabilistic methods in approximation theory: a general setting, Atti Sem. Mat. Fis. Univ. Modena 40 (1992), no. 1, 137-147.

[12] J. de la Cal and A. M. Valle, Global smoothness preservation by multivariate Bernstein-type operators, Handbook of Analytic-Computational Methods in Applied Mathematics (G. Anastassiou, ed.), Chapman \& Hall/CRC, Florida, 2000, pp. 667-707.

[13] R. A. DeVore, The Approximation of Continuous Functions by Positive Linear Operators, Lecture Notes in Mathematics, vol. 293, Springer-Verlag, Berlin, 1972.

[14] R. A. DeVore and G. G. Lorentz, Constructive Approximation, Grundlehren der Mathematischen Wissenschaften, vol. 303, Springer-Verlag, Berlin, 1993.

[15] Z. Ditzian and V. Totik, Moduli of Smoothness, Springer Series in Computational Mathematics, vol. 9, Springer-Verlag, New York, 1987.

[16] B. Fuchssteiner, Daniell lattices and adapted cones, Arch. Math. (Basel) 37 (1981), no. 6, 528-532.

[17] L. Gillman and M. Jerison, Rings of Continuous Functions, Graduate Texts in Mathematics, no. 43, Springer-Verlag, New York, 1976.

[18] H. H. Gonska, The rate of convergence of bounded linear processes on spaces of continuous functions, Automat. Comput. Appl. Math. 7 (1998), no. 1, 3897.

[19] M. M. A. Jimenez Pozo, Sur les opérateurs linéaires positifs et la méthode des fonctions tests, C. R. Acad. Sci. Paris Sér. A 278 (1974), 149-152 (French).

[20] B. J. P. King, The Lototsky transform and Bernstein polynomials, Canad. J. Math. 18 (1966), 89-91.

[21] E. M. Mangino, Differential operators with second-order degeneracy and positive approximation processes, Constr. Approx. 18 (2002), no. 3, 443-466.

[22] _ A positive approximation sequence related to Black and Scholes equation, Rend. Circ. Mat. Palermo (2) Suppl. 68 (2002), 657-670.

[23] T. Nishishiraho, The degree of convergence of positive linear operators, Tohoku Math. J. (2) 29 (1977), no. 1, 81-89.

[24] _ Quantitative theorems on approximation processes of positive linear operators, Multivariate Approximation Theory, II (Oberwolfach, 1982) (W. Schempp and K. Zeller, eds.), Internat. Ser. Numer. Math., vol. 61, Birkhäuser Verlag, Basel, 1982, pp. 297-311.

[25] _ The order of approximation by positive linear operators, Tohoku Math. J. (2) 40 (1988), no. 4, 617-632. 
Francesco Altomare: Department of Mathematics, University of Bari, Via Orabona 4, 70125 Bari, Italy

E-mail address: altomare@dm. uniba.it

Sabrina Diomede: Department of Economic Sciences, University of Bari, Via C. Rosalba 53, 70124 Bari, Italy

E-mail address: s.diomede@dse.uniba. it 


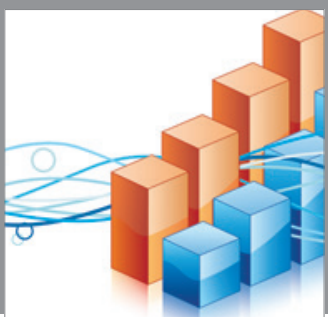

Advances in

Operations Research

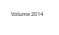

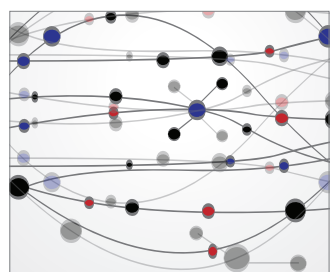

\section{The Scientific} World Journal
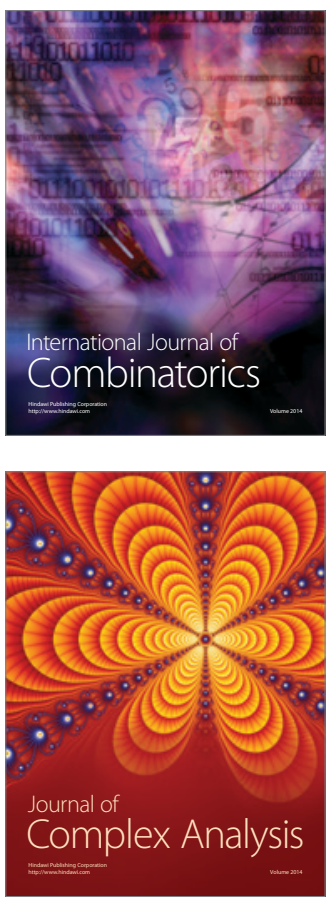

International Journal of

Mathematics and

Mathematical

Sciences
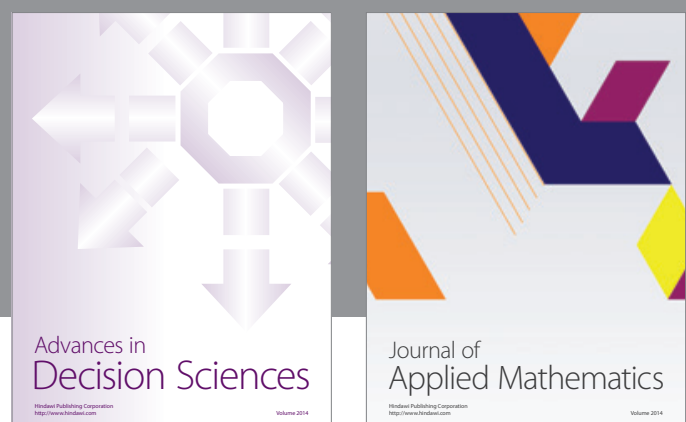

Journal of

Applied Mathematics
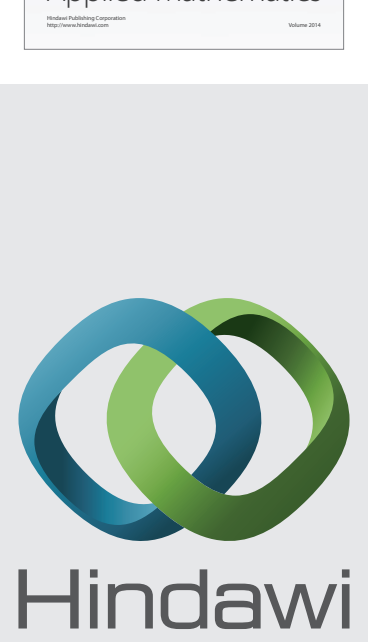

Submit your manuscripts at http://www.hindawi.com
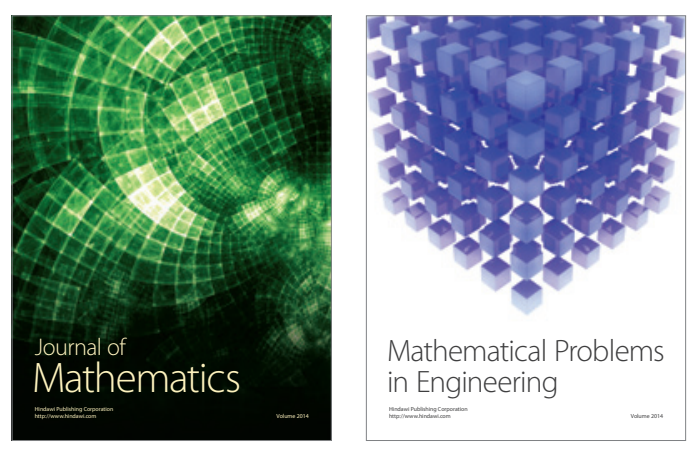

Mathematical Problems in Engineering
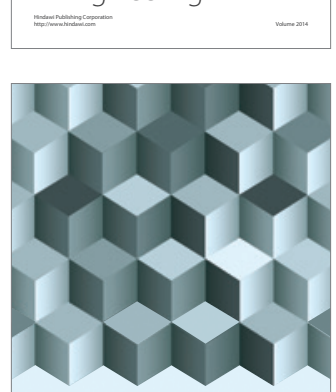

Journal of

Function Spaces
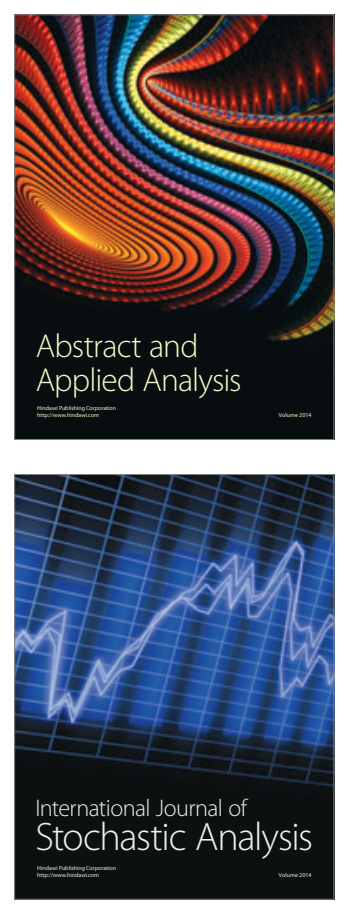

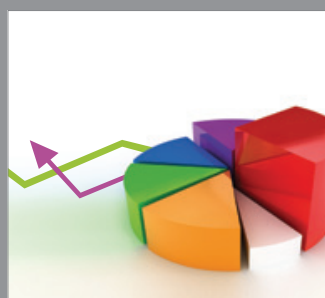

ournal of

Probability and Statistics

Promensencen
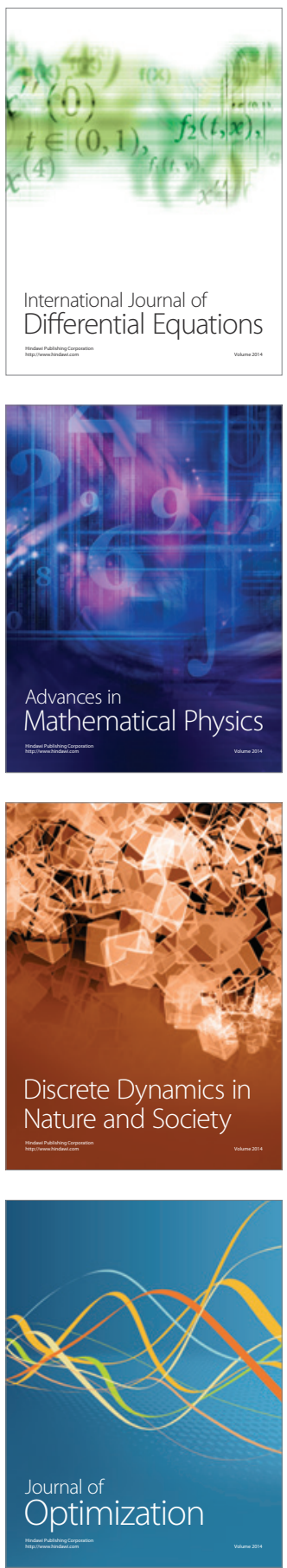